\title{
Comparative Genomics, Pangenome, and Phylogenomic Analyses of Brenneria spp., and Delineation of Brenneria izadpanahii sp. nov.
}

\author{
Meysam Bakhshi ganje, ${ }^{1}$ John Mackay, ${ }^{2}$ Mogens Nicolaisen, ${ }^{3}$ and Masoud Shams-Bakhsh ${ }^{1, \dagger}$ \\ ${ }^{1}$ Department of Plant Pathology, Faculty of Agriculture, Tarbiat Modares University, Tehran, Iran \\ 2 Department of Plant Sciences, University of Oxford, Oxford, U.K. \\ ${ }^{3}$ Faculty of Science and Technology, Department of Agroecology, Aarhus University, Forsøgsvej 1, 4200, Slagelse, Denmark \\ Accepted for publication 11 May 2020.
}

\begin{abstract}
Brenneria species are bacterial plant pathogens mainly affecting woody plants. Association of all members with devastating disorders (e.g., acute oak decline in Iran and United Kingdom) are due to adaptation and pathogenic behavior in response to host and environmental factors. Some species, including B. goodwinii, B. salicis, and B. nigrifluens, also show endophytic residence. Here we show that all species including novel Brenneria sp. are closely related. Gene-based and genome/pangenomebased phylogeny divide the genus into two distinct lineages, Brenneria clades $\mathrm{A}$ and $\mathrm{B}$. The two clades were functionally distinct and were consistent with their common and special potential activities as determined

B. corticis. An extensive repertoire of candidate virulence factors was identified. Comparative genomics indicated a repertoire of plant cell wall degrading enzymes, metabolites/antibiotics, and numerous prophages providing new insights into Brenneria-host interactions and appropriate targets for further characterization. This work not only documented the genetic differentiation of Brenneria species but also delineates a more functionally driven understanding of Brenneria by comparison with relevant Pectobacteriaceae thereby substantially enriching the extent of information available for functional genomic investigations.
\end{abstract} via annotation of functional domains. Pangenome analysis demonstrated that the core pathogenicity factors were highly conserved, an hrp gene cluster encoding a type III secretion system was found in all species except
Keywords: Brenneria izadpanahii, Brenneria spp., comparative genomics, pangenome analysis, phylogenomics
The family Pectobacteriaceae has become a major threat to trees worldwide. Most species from this genus seem ubiquitous and were isolated from a variety of distinctive forest habitats and urban trees (Brady et al. 2014; Denman et al. 2012; Hauben et al. 1998; Li et al. 2015, 2019; Zheng et al. 2017). The destructive capability and a causal association of Brenneria spp. with diseased woody plants have been demonstrated (Broberg et al. 2018; Hauben et al. 1998). Due to their wide spatiotemporal distribution and diverse habitats (Maes et al. 2009; Pettifor et al. 2020), the range of tree species affected and the severe pathogenic activity in the microbiota (e.g., acute oak decline [AOD] microbiome), their impacts can be devastating on forest health (Broberg et al. 2018; Sapp et al. 2016). Research aimed at deciphering their pathogenic behavior within their ecological niches has become crucial to shed light onto their ecological roles and their pathogenic activity.

At the time of writing, the genus Brenneria comprises eight species with valid published names (www.bacterio.net/brenneria.html): B. alni, B. salicis, B. goodwinii, B. nigrifluens, B. rubrifaciens, B. roseae, B. corticis, and B. populi (Brady et al. 2014; Denman et al. 2012; Hauben et al. 1998; Li et al. 2015, 2019). Molecular markerbased taxonomy paradigms have changed with the transition from multilocus sequence analysis and typing (MLSA/MLST) 16S rRNA to genome-based phylogeny, with many Brenneria spp. strains being assigned into novel taxa as it was shown that they have been misidentified based on phenotypic-genotypic features. Adeolu et al. (2016) reconstructed phylogenetic and taxonomic relationships in Enterobacteriales and introduced the

†Corresponding author: M. Shams-Bakhsh; shamsbakhsh@modares.ac.ir

Funding: Financial support provided by Tarbiat Modares University.

The author(s) declare no conflict of interest.

(C) 2021 The American Phytopathological Society monophyletic Dickeya-Pectobacterium (Brenneria) clade based on a comprehensive study using genomic data. The relationship among Brenneria species is not completely clear because phylogenetic trees were based on MLSA of only four housekeeping genes at most and genome-based phylogenetic analyses were conducted only with a few species (Denman et al. 2012; Zhang et al. 2016). Given their phytopathological importance, the number of available Brenneria spp. genome sequences in GenBank has increased recently; however, their taxonomic placement are still controversial and additional investigations are needed to further clarify the taxonomy of the genus.

High throughput sequencing has produced bacterial genome datasets enabling comparative genomic analyses and facilitating wider and more comprehensive assessments of similarities and differences between and within plant and animal pathogens (Toth et al. 2006). Doonan et al. (2019) demonstrated that canonical bacterial species contributed to the AOD syndrome by using comparative genomics and showed that orthologous gene inferences (e.g., identification of virulence gene orthogroups) can be used to evaluate the pathogenic potential of bacterial species and their impacts on host-microbe and microbe-microbe interactions. They identified B. goodwinii as a keystone taxa and primary pathogen that contributes vital pathogenicity repertoires in the polymicrobial consortium of AOD causal agents (Doonan et al. 2019). The communications among the community of species associated with the AOD, the interactions with other symbionts in the environment (B. goodwinii and Gibbsiella quercinecans in AOD syndrome), and the potential mechanisms and effects of Brenneria species on related pathobiome are diverse and incompletely understood.

Despite the deep knowledge of the soft rot Pectobacteriaceae (Pectobacterium spp. and Dickeya spp.), genomic information of other members like Brenneria and Lonsdaleae (formerly Brenneria) is still scarce. Our objectives were to (i) conduct a comparative genomics investigation among Brenneria spp. using all available 
genome sequences; (ii) develop a novel taxonomic understanding of the genus; and (iii) clarify whether we have uncovered a new species of Brenneria in the north of Iran, represented by a Brenneria sp. isolate obtained from diseased oak trees. To this end, we inferred the phylogeny of the novel isolate and other strains of the family Pectobacteriaceae. A pangenome analysis emphasizing the predicted pathogenicity determinants was obtained by considering an increasing number of bacterial species, most of which represent new microflora and lack of a holistic comparison.

\section{MATERIALS AND METHODS}

Maintenance of bacterial strain, 16S rRNA/MLSA typing, genomic DNA extraction, library preparation, and genome sequencing. The novel isolate of Brenneria sp. (Iran isolate 50) was obtained from diseased oak trees (Quercus castaneifolia) in the north of Iran. It was stored in $40 \%$ glycerol stocks at $-80^{\circ} \mathrm{C}$ and maintained on nutrient agar at $30^{\circ} \mathrm{C}$. Genomic DNA for amplification was extracted by the alkali extraction method (Niemann et al. 1997) and stored at $-20^{\circ} \mathrm{C}$ until use. The 16S rRNA gene and MLSA (for housekeeping genes $\operatorname{gy} B$, infB, and $a t p D$ ) sequencing were conducted using primers and sequencing conditions according to Hauben et al. (1998) and Brady et al. (2008), respectively. The 16S rRNA gene and MLSA similarities evaluation were conducted by the EzTaxon-e (https://www.ezbiocloud.net/identify) for 16S rRNA gene in special and GenBank similarity engine (Blastn) for both. High-quality genomic DNA was extracted using Qiagen Genomic-tip 500/G following the manufacturer's instructions (Qiagen, Germany). Genome sequencing of the novel isolate was accomplished with single molecule real-time (SMRT) technology of the Pacific Biosciences RSII platform (Macrogen Co., South Korea). The library was reconstructed with approximately 20-kb insert size using the SMRT Cell 8Pac V3, DNA Polymerase Binding Kit P6, DNA Sequencing Reagent 4.0 v2. The quality (DNA integrity) and quantity of the extracted genome and subsequent library were evaluated using $1 \%$ agarose gel electrophoresis and spectrophotometry/Nanodrop/DNA Bioanalyzer 12000 chip and DNA QC-Picogreen, respectively.

Genome assembly of pacific biosciences RSII generated data. The de novo sequence assembly was performed using the hierarchical genome assembly 3 (HGAP3) workflow, incorporating the CELERA assembler and finished using the Quiver consensus polisher according to Doonan et al. (2019). Genome estimated completeness and contamination were verified with CheckM version 1.0.7 (Parks et al. 2015). Resultant assembly produced one contig and $\mathrm{a}<200 \times$ sequencing depth.

Additional whole genome sequence data. Twenty-four draft genome sequences consisting of all valid published genomes from genus Brenneria (January 2020) and a few genomes from Lonsdalea spp., P. carotovorum ssp. carotovorum, and Dickeya spp. were retrieved from the NCBI GenBank database (Table 1). These genomes were incorporated into the workflow described below.

Genome annotation, identification of orthologs, and pangenome analysis. Aiming to assess the level of discrepancy among the genomes, whole genome sequence identity was computed using indices useful for species delineation; pairwise average nucleotide identity (ANI) according to BLAST (ANIb) in JSpeciesWS (Richter et al. 2016); ANI calculator (https:// www.ezbiocloud.net/tools/ani) (Yoon et al. 2017) and OrthoANI values with the standalone orthologous average nucleotide identity tool (OAT) in Ezbiocloud (Lee et al. 2016). Also, digital DNADNA hybridization $(\mathrm{dDDH})$ percentages were determined in silico with the genome-to-genome distance calculator (dGGDC 2.0) using the BLAST method and recommended formula 2 (MeierKolthoff et al. 2013) (http://ggdc.dsmz.de/ggdc.php\#). Both ANI and dDDH values were below the accepted threshold in the case of the new species ( $\leq 95$ and $\leq 70 \%$ for ANI and dDDH, respectively) (Table 2).
Automatic annotation of draft genomes and complete genome of Brenneria sp. achieved in this study was conducted using RAST (Overbeek et al. 2014). Also, prokaryotic genome assembly and annotation were performed using KBase, according to Arkin et al. (2018). New raw/annotated genomes (e.g., B. corticis) were imported into the KBase. A genome set was made for all genomes and then phylogenetic trees were constructed from the Brenneria sp. along with the 29 nearest neighbors using the Insert Genome into Species Tree app (version 2.1.10), which utilizes FastTree 2 (Price et al. 2010). A pangenome was constructed Build Pangenome with OrthoMCL app (Pangenome Orthomcl v0.0.7) using aforementioned genome set. Pangenomes were visualized using the Pangenome Circle Plot app (v1.2.0) and pangenome-based phylogenomic analysis was conducted by the Phylogenetic Pangenome Accumulation (v1.4.0) app. Genomes were further compared by annotating the functional domains in the whole genome set with DomainAnnotation (version 1.03) and viewing the results using the View Function Profile for Genomes app (version 1.0.1) with the domain namespace set to BSEED Roles.

The online bacterial genome analysis service PATRIC (Pathosystems Resource Integration Center) was used for determination of the GC content, number of coding sequence regions (CDS), tRNA genes, pseudogenes, and others features, and the obtained information was used to reconstruct metabolic networks and subsystems. The clusters that were involved in diverse categories such as virulence and pathogenicity determinants, including type secretion systems (TSS), phytotoxins, iron uptake, polysaccharides biosynthesis, flagella encoding genes, and cell attachment were screened and compared across all Brenneria species. Circular genome maps were created using the circus software in PATRIC (Wattam et al. 2017). Also, the online service IslandViewer version 4.0 (an integrated interface for computational identification and visualization of genomic islands; http://www.pathogenomics.sfu. ca/islandviewer) was used for identification of pathogenicity islands within our bacterial genome (Bertelli et al. 2017).

The CAZy and dbCAN2 meta server databases were used to annotate plant cell wall degrading enzymes (PCWDEs) (Lombard et al. 2014; Zhang et al. 2018). The presence of plasmids was screened using PlasmidFinder 2.0 (https://cge.cbs.dtu.dk/services/ PlasmidFinder/) (Carattoli et al. 2014) for all the genomic sequences. The online service PHASTER (PHAge Search Tool Enhanced Release; http://phaster.ca/) was used for identification of prophage sequences within bacterial genomes (Arndt et al. 2016). The Prokka annotation output was used once as input data for in silico secondary metabolite and antibiotic profiling by searching against the antiSMASH database-bacterial version (https://antismash.secondarymetabolites.org/\#!/start) (Blin et al. 2019) in addition to the genome wide comparisons and visualization of orthologous clusters that were performed using the online service OrthoVenn2 (https://orthovenn2.bioinfotoolkits.net/) (Xu et al. 2019).

\section{RESULTS}

Phylogenetic analysis, genomic DNA extraction, and qualification of library preparation. Concatenated phylogenetic trees placed Brenneria sp. from this study separately from other Brenneria clade members, indicating that the isolate from the north of Iran likely belongs to a novel species in the genus Brenneria. Sequence similarity assessments using 16S rRNA gene and MLSA ( $g y r B$, infB, and $a t p D$ ) showed that the novel Brenneria sp. is most similar ( 97 to $98 \%$ ) to B. goodwinii along with all other Brenneria species (Fig. 1A). The quantification and qualification of produced library demonstrated the insert size was almost $17 \mathrm{~kb}$.

Complete genome sequencing of $B$. izadpanahii sp. nov. The sequence data for B. izadpanahii (isolated from diseased oak trees at the north of Iran) was assembled into one contig and the genome size was determined to be $5,330,697$ bp by using the hierarchical genome assembly 3 (HGAP3) workflow. Annotation 
of the complete genome predicted 4,743 CDS transcribed for B. izadpanahii. It identified 39 rRNA, 255 tRNA, 153 ribosomal protein, and 79 flagellum and flagellar motility-related genes. Subsystems and their related gene categories (e.g., metabolism), circular view of annotated subsystems within the B. izadpanahii genome, and pathogenicity islands screened by using IslandViewer tool are depicted in Figure 2A, B, and C, respectively.

The ANI/ANIb/orthoANI and dDDH values between B. izadpanahii and other Pectobacteriaceae members were estimated. The ANI values varied from 78 to $90 \%$ between different pairs of strains, showing B. goodwinii strain 141 with (89.98/90.35/90.57-41.10) identity/similarity lower than the proposed species boundary ANI cut-off value of 95 to $96 \%$ and $\leq 70 \%$ for dDDH was the most similar neighbor (Table 2).

Whole genome phylogeny. All Brenneria clade members appeared to be closely related and the whole genome phylogeny suggests two distinct lineages (Fig. 1B). Clade A has five taxa including B. goodwinii, B. izadpanahii, B. alni, B. nigrifluens, and $B$. corticis that appear to share a recent common ancestor. Clade $B$ includes the three others, B. rosease, B. rubrifaciens and B. salicis, sharing another recent common ancestor. The phylogeny also indicates that the Brenneria clades share a common recent ancestor with the Pectobacterium spp. and are more distantly related to Dickeya spp. and Lonsdalea spp. and share least with the Sodalia ssp. These results are in agreement with the rearrangements of Enterobacteriales and creation of the family Pectobacteriaceae (Adeolu et al. 2016).
Pangenome analysis. The pangenome comparisons of Brenneria clades A and B and other relevant in Pectobacteriaceae indicated the distinctive status and genetic makeup of B. izadpanahii (Figs. 1C and 3). Summary statistics of the pangenome datasets and shared genes are presented in Table 3. Genomes in Brenneria clades (A and B) had a total of 47,402 genes. Of the genes in clade A, 28,745 are in homologous families among the pangenome, and 2,680 are in singleton families; while in clade B, 13,547 genes are in homologous families and 2,430 are in singleton families.

Orthologous protein families and clusters were identified and categorized in both KBase and Orthovenn 2 and were visualized by online web server Orthovenn 2 for genome sets. Comparisons of the orthologous gene clusters within species of clades A and B and other relevant were determined through four versus four, five versus five, and six versus six genome sets (Fig. 4).

Brenneria clade A and B species shared 2,391 and 2,369 proteins in their genome sequences, respectively (Fig. 4A and C). Clade A members (B. izadpanahii, B. goodwinii, B. alni, B. nigrifluens, and B. corticis) showed 534, 365, 438, 377, and 443 unique proteins among their genome sequences, and clade B (B. salicis, B. rubrifaciens, $B$. roseae ssp. americana, and B. roseae ssp. roseae) had 526, 292, 275, and 251 unique proteins, respectively. Similarities and relatedness of the orthologous clusters within the genomes were depicted as a heatmap (Fig. 5B). As expected in clades A and B phylogeny, B. izadpanahii-B. goodwinii, B. nigrifluens-B. corticis, and B. roseae ssp. americana- $B$. roseae ssp. roseae had most similarities.

TABLE 1. Genome metrics of Brenneria izadpanahii sp. nov. and other genomes available in GenBank database used in this study

\begin{tabular}{|c|c|c|c|c|c|}
\hline Organism (accession) & Origin of isolation & $\begin{array}{l}\text { Number of } \\
\text { contigs }\end{array}$ & $\begin{array}{c}\text { Chromosome } \\
\text { size }(\mathrm{bp}) / \mathrm{GC} \\
\text { content }(\mathrm{mol} \%)\end{array}$ & $\begin{array}{l}\text { Number of } \\
\text { coding sequence } \\
\text { regions }\end{array}$ & Reference \\
\hline B. izadpanahii Iran 50 (CP050854) & Mazandaran, Iran & 1 & $5,330,697(53.7)$ & 4,743 & This study \\
\hline B. goodwinii FRB141 (CP014137) & Outwood, U.K. & 1 & $5,281,917(53.2)$ & 4,625 & Doonan et al. (2019) \\
\hline B. goodwinii FRB171 (MJLY00000000) & Gorse Covert, U.K. & 128 & $5,377,922(53.0)$ & 4,881 & Doonan et al. (2019) \\
\hline B. goodwinii OBR1 (CGIG00000000) & U.K. & 1 & $5,350,059(53.1)$ & 4,835 & Doonan et al. (2019) \\
\hline $\begin{array}{l}\text { B. nigrifluens ATCC } 13028 \\
\text { (CP034036) }\end{array}$ & California, U.S.A. & 1 & $4,891,702(55.9)$ & 4,392 & $\begin{array}{l}\text { Poret-Peterson et al. } \\
\qquad(2019)\end{array}$ \\
\hline $\begin{array}{l}\text { B. nigrifluens LMG } 2694 \\
\text { (QDKK00000000) }\end{array}$ & - & 84 & 4,837,857 (55.9) & 4,395 & Li et al. (2019) \\
\hline $\begin{array}{l}\text { Brenneria sp. EniD312 } \\
(\text { NZ_CM001230.1) }\end{array}$ & - & 1 & $4,943,773(55.9)$ & 4,540 & - \\
\hline B. alni NCPPB 3934 (MJLZ00000000) & Italy & 132 & $4,127,267(51.1)$ & 4,013 & Doonan et al. (2019) \\
\hline $\begin{array}{l}\text { B. alni DSM11811 (BioProject } \\
\text { PRJNA234905) }\end{array}$ & - & 60 & $4,100,984(51)$ & 3,803 & - \\
\hline B. salicis DSM30166 (MJMA00000000) & - & 106 & $3,929,937(52.1)$ & 3,781 & Doonan et al. (2019) \\
\hline B. salicis (NZ_QNRY01000001) & - & 93 & $3,907,359(52.06)$ & 3,613 & - \\
\hline B. rubrifaciens 6D370 (CP034035) & California, U.S.A. & 1 & $4,028,093(52.6)$ & 3,521 & $\begin{array}{l}\text { Poret-Peterson et al. } \\
\qquad(2019)\end{array}$ \\
\hline $\begin{array}{l}\text { B. roseae ssp. americana LMG } 27715 \\
\text { (NZ_QDKJ00000000.1) }\end{array}$ & U.K. & 54 & $4,476,002(51.7)$ & 4,152 & Li et al. (2019 \\
\hline $\begin{array}{l}\text { B. roseae ssp. roseae LMG } 27714 \\
\text { (QDKI00000000) }\end{array}$ & U.K. & 58 & $4,504,465(51.6)$ & 4,196 & Li et al. (2019) \\
\hline $\begin{array}{l}\text { B. corticis } \mathrm{gBX} 10-1-2 \\
(\mathrm{QDKH} 00000000)\end{array}$ & $\begin{array}{l}\text { Shandong and Henan, } \\
\text { China }\end{array}$ & 56 & $5,040,874(56.4)$ & 4,501 & Li et al. (2019) \\
\hline $\begin{array}{l}\text { Lonsdalea quercina ssp. quercina ATCC } \\
29281 \text { (NZ_JIBO00000000.1) }\end{array}$ & U.S.A. & 37 & $3,850,073(55.1)$ & 3,327 & Caballero et al. (2014) \\
\hline $\begin{array}{l}\text { L. quercina ssp. iberica LMG } 26265 \\
\text { (NZ_LUTQ00000000.1) }\end{array}$ & - & 150 & $3,755,411(55.04)$ & 3,362 & - \\
\hline $\begin{array}{l}\text { L. quercina ssp. britannica } 477 \\
(\mathrm{CP} 023009)\end{array}$ & Surrey, U.K. & 1 & $4,015,589(55.1)$ & 3,801 & Doonan et al. (2019) \\
\hline $\begin{array}{l}\text { L. quercina ssp. britannica LMG } 26267 \\
\text { (NZ_LUTN00000000.1) }\end{array}$ & - & 57 & $3,882,080(55.1)$ & 3,475 & - \\
\hline $\begin{array}{l}\text { L. quercina ssp. populi CFCC } 11196 \\
\text { (NZ_LUTE00000000.1) }\end{array}$ & - & 130 & $3,784,815(55.4)$ & 3,381 & - \\
\hline $\begin{array}{l}\text { L. quercina ssp. populi CFCC13122 } \\
\text { (NZ_LUTI01000001) }\end{array}$ & - & 163 & $3,648,192(55.4)$ & 3,304 & - \\
\hline Dickeya dadantii 3937 (NC_014500) & - & 1 & $4,922,802(56.3)$ & 4,513 & Glasner et al. (2011) \\
\hline $\begin{array}{l}\text { D. paradisaica NCPPB } 2511 \\
\text { (NZ_CM001857) }\end{array}$ & Colombia & 1 & $4,631,867(55.0)$ & 4,016 & Pritchard et al. (2013) \\
\hline $\begin{array}{l}\text { Pectobacterium carotovorum ssp. } \\
\text { carotovorum PC1 (NC_012917) }\end{array}$ & - & 1 & $4,862,913(51.9)$ & 4,461 & - \\
\hline
\end{tabular}


The orthoANI diagram from OTA tool and the distance matrices heatmap of the generated orthologous clusters from Orthovenn 2 showed highly consistent results although they are nucleotide and protein-based pipelines, respectively (Fig. 5A and B).

The clades identified in the phylogenetic analysis were also functionally distinct as determined via annotation of functional domains in the genomes using the SEED database. Genes in all categories (especially in the singletons) that were compared between the genomes included those specifically associated with transcription regulators, potentially associated virulence genes and carbohydrate, amino acid and lipid transport, and metabolism (Fig. 6). In comparison between B. izadpanahii and its closest neighbor, B. goodwinii 141, unique pathogenicity associated domains was notable such as transcriptional regulator, AraC family, type IV secretory pathway, VirD4 components, and many phage-related domains in B. izadpanahii and B. goodwinii 141, respectively.

Pathogenicity gene clusters in Brenneria clades. Similar to other necrotrophic phytopathogens, pathogenicity determinants within the genus Brenneria including bacterial secretion systems are highly conserved (Doonan et al. 2019). These systems include PCWDEs, which may have a crucial role in disease occurrence, progress and suppression of the plant defense mechanisms. Sequence similarity searches may be employed in order to determine putative pathogenicity mechanisms in novel bacterial pathogens by identifying the core repertoire of virulence genes shared by related organisms (Broberg et al. 2018; Doonan et al. 2019). Significant potential virulence homologs were determined in silico in Brenneria clade A and B species and compared with other relevant Pectobacteriaceae emphasizing PCWDEs (particularly pectinases), functional secretion systems (in special type III T3SS), and associated effectors and harpins.

We identified PCWDEs by using automated CAZym annotation performed in dbCAN2 meta-server database, which identifies closely related carbohydrate active enzymes including PCWDEs. The annotation results revealed that the family Pectobacteriaceae has a core enzyme repertoire in addition to a few specialists that might be involved in interactions with host plants and other

TABLE 2. Definition of the in silico average nucleotide identity and DNA-DNA hybridization values in the form (ANI/ANIb/orthoANI-dDDH) (first column only), ANI index for the rest of columns, of Brenneria izadpanahii sp. nov. and representative strains of the other described Pectobacteriaceae species ${ }^{\mathrm{a}}$

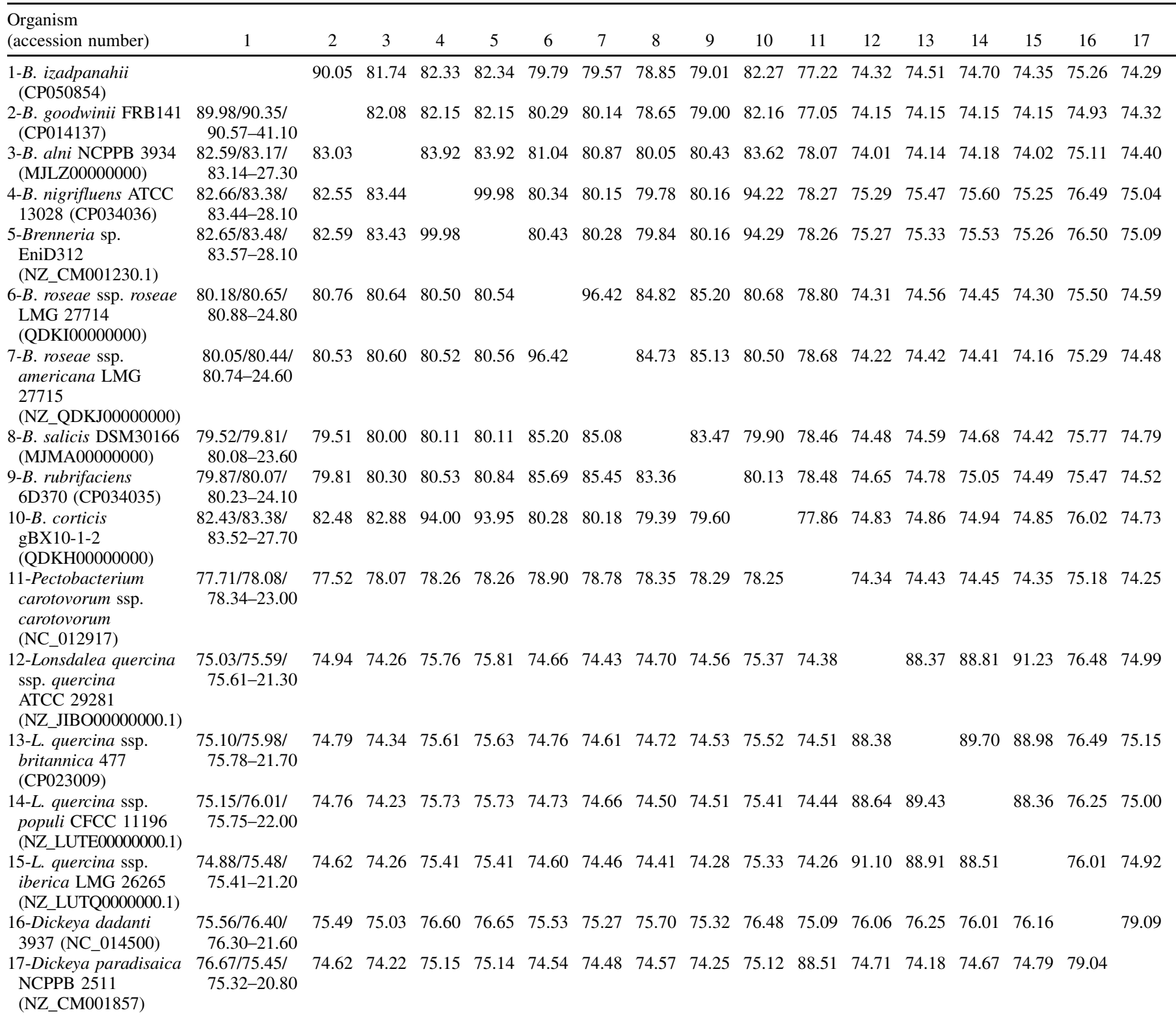

a The specific threshold value is 95 to $96 \%$ for ANI and $70 \%$ for DDH. ANI values were calculated using three different algorithms, i.e., ANI calculator, JSpeciesWS (ANIb), and OrthoANI. 
symbionts (Table 4). The most common enzymes we identified were annotated as polysaccharide lyase (PL) family, PL22 (oligogalacturonide lyase [EC 4.2.2.6]); glycoside hydrolases (GH) family, GH102 (peptidoglycan lytic transglycosylase [EC
3.2.1.-]); and glycosyl transferase (GT) family, GT2 — with variety of activities - were notable. Also, the family carbohydrate esterase (CE) only had three most frequent and top hits for CE1 in Dickeya dadantii, D. paradisaica, and $P$. carotovorum ssp. carotovorum
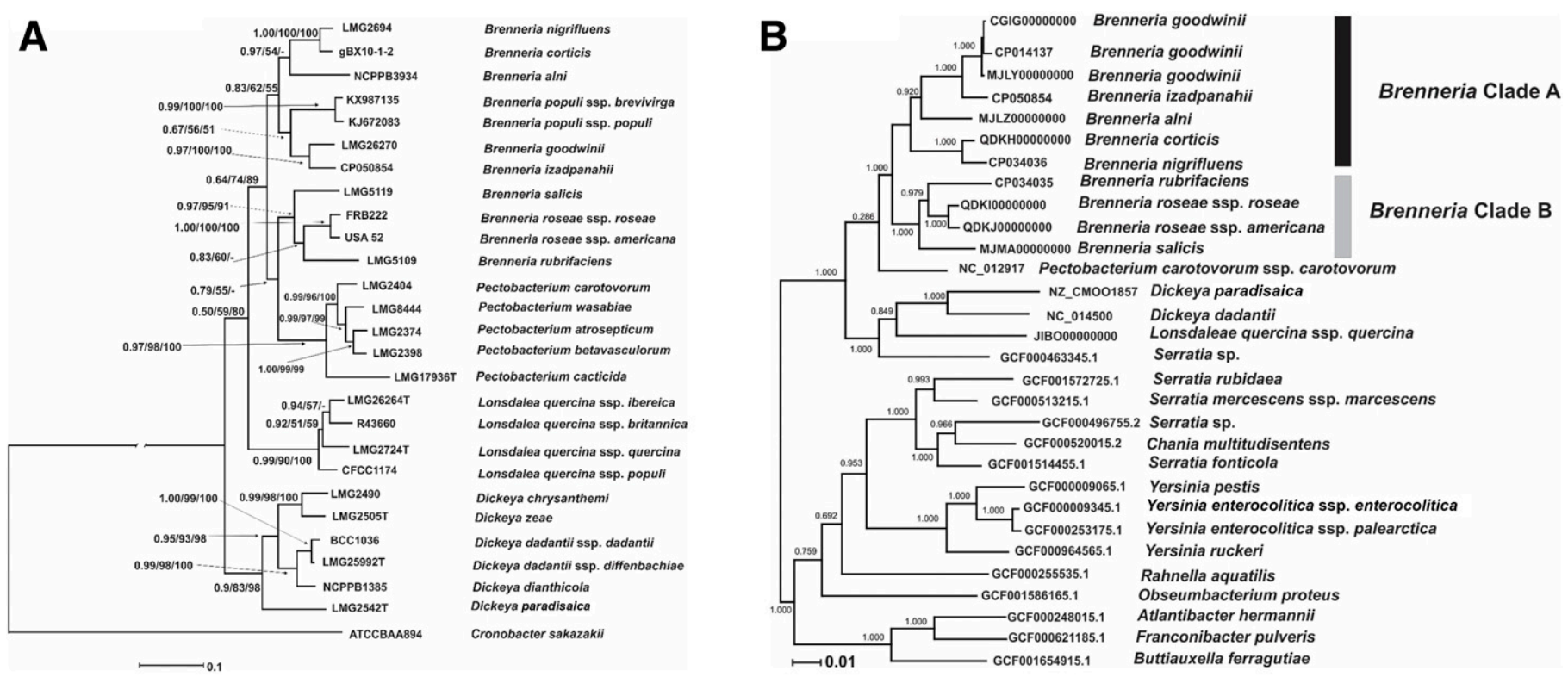

\section{C}
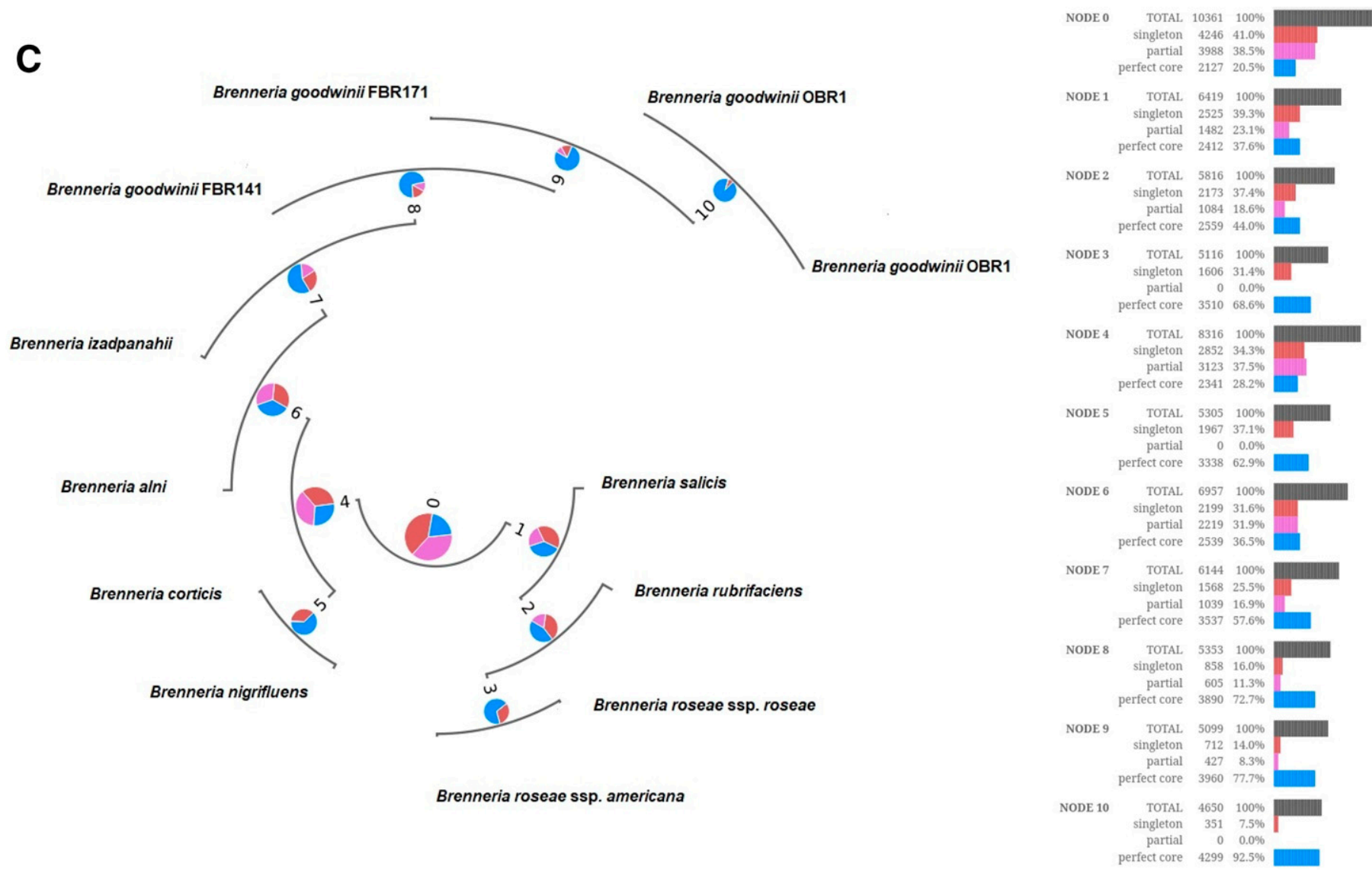

Fig. 1. A, Bayesian 50\% majority rule consensus tree based on concatenated partial $16 S$ rRNA/gyrB, atpD, and infB gene sequences of Brenneria izadpanahii sp. nov. and all validly described species of the genus Brenneria in addition to phylogenetically related species under the GTR $+\mathrm{G}+\mathrm{I}$ model. Bayesian posterior probabilities (BPP), maximum likelihood bootstrap (ML BS), and maximum parsimony bootstrap (MP BS) values $>50 \%$ are given for appropriate clades in the form: BPP/ML/MP BS. Cronobacter sakazakii is included as an out-group. The scale bar indicates the fraction of substitutions per site. B, Phylogenomic tree of the Brenneria clade A and B species along with neighbor Pectobacteriaceae members generated by Insert Genome into Species Tree app in KBase using the 29 closest relatives in the public database. Numbers are local support values. C, Pangenome-based phylogenomic analysis of Brenneria clade A and B species. Orthologous gene set percentages within a pangenome partitioned into three categories: core (blue), singleton (red), and partial pangenome (pink). Pangenome created by OrthoMCL app distinguish which homologous genes are true orthologs and vertically inherited from those with lineage derived paralogous expansions by duplication. 
PC1 in genome set. A pectate lyase, PL4 (EC 4.2.2.23), which degrades the plant cell wall component rhamnogalacturonan, was determined uniquely in B. izadpanahii and B. goodwinii (Table 4).

The T3SS is essential to the virulence of numerous phytopathogens, e.g., Pseudomonas syringae pathovars that use the system to enable host manipulation (Tampakaki et al. 2010). In Brenneria clade A, complete T3SS was detected in all members but surprisingly typical genes in the core type III nanomachinery were not identified in the B. corticis genome; the result was confirmed with the analysis system in PATRIC.

Pathogenicity islands encoding the secretion of effector molecules via type III secretion system have been discovered in several gram-negative pathogens (Winstanley and Hart 2001). The hrpN harpin gene is key to the virulence of E. amylovora and secretes the DspA/E effector, both $h r p N$ and $d s p A / E$ are encoded within Brenneria species. (Doonan et al. 2019). HrpG, HrpD, HrpA (TTSS pilin hrpA), HrpF, HrpW (harpin with pectate lyase domain), HrpB, HrpP (annotated as hypothetical protein in some cases), HrpQ, and HrpJ were found in all clade A and B members, except for B. corticis, which only shares both ATP-dependent helicase HrpA and ATP-dependent helicase HrpB with other members. Type III secretion protein HrpT (annotated as hypothetical protein in some cases) was found in all members except for B. alni, B. cortices, and B. nigrifluens.

As described previously in AOD pathobiome, the major $B$. goodwinii homologous virulence factors, effector repertoire, were screened and hopAN1, hopX1, hopAJ2, hopA1, hopAW1, avrXccB, $d s p A / E, d s p F, m x i E$, and $a v r A x v$, which are overall key to disease causation in bacterial phytopathogens, were detected (Doonan et al. 2019). We identified potential virulence factors to add to those of Doonan et al. (2019); they include hopVl (B. alni), virulence factor VirK (B. goodwinii and B. izadpanahii), and protein of avirulence locus impE (B. izadpanahii) in Brenneria clade A species.
In Brenneria clade B, homologous sequences were identified for virulence factors such as type III effector protein AvrE1 (B. roseae ssp. roseae, B. roseae ssp. americana, and $B$. salicis, annotated as hypothetical protein in $B$. rubrifaciens) and hopVl within the $B$. salicis and $B$. rubrifaciens genomes. Sequences detected in both clades A and B of Brenneria included virulence factor MviM (B. salicis, B. goodwinii 141/171/OBR, B. izadpanahii, and B. roseae ssp. americana), virulence-associated protein VagC (B. corticis; $B$. roseae ssp. roseae), and virulence-associated protein $\mathrm{C}$ (VapC toxin protein) (B. corticis-two families, B. nigrifluens, B. roseae ssp. roseae, and $B$. roseae ssp. americana).

In silico detection of the plasmids, phages, and secondary metabolites. The genome datasets were scanned for plasmids by the PlasmidFinder online service for Brenneria clades A and B and of relevant Pectobacteriaceae members, and the results indicated that they carry no detectable plasmid.

Scanning the Brenneria spp. genomes investigated in this study for prophage sequences with the PHASTER online service detected several hypothetical prophage groups including PHAGE_Salmon_SEN1_NC_029003(3), PHAGE_Aeromo_ phiO18P_NC_009542(16), and PHAGE_Gordon_Schwabeltier_ NC_031255(1) (Table 5). We detected a few additional phage regions by using a GenBank formatted file (.gff) as a query that were not found when using FASTA formatted genomes files. Also, in our experience, the number of genomes contigs in .gff format could affect outputs. For example, using FASTA drafted genome of $B$. corticis, three prophage groups were determined, but using. gff format at least seven different prophage families were detected within the genome.

Bacterial metabolite detection online tools were developed reinforcing the importance of their biological and ecological functions. Turnurbactin (up to $46 \%$ similarity with the reference data-Teredinibacter turnerae T7901-GenBank CP001614.2) and aryl polyene $(100 \%$ similarity-Xenorhabdus doucetiae strain FRM16, reference sequence NZ_FO704550.1) were detected as

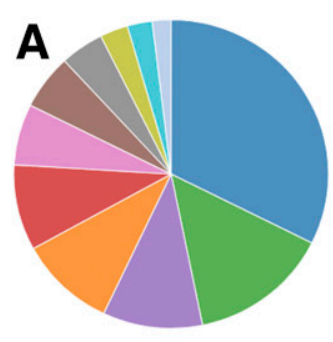

Subsystem (Subsystems, Genes)
- METABOLISM (101, 895)
PROTEIN PROCESSING $(45,265)$ - PROTEIN PROCESSING $(45,265)$ INERGY $(31,269)$ - MEMBRANE TRANSPORT $(28,214)$ DNA PROCESSING $(20,110)$ I CELLULAR PROCESSES $(18,118)$ - RNA PROCESSING $(14,73)$ a CELL ENVELOPE $(9,71)$ REGULATION AND CELL SIGNALING $(6,20)$

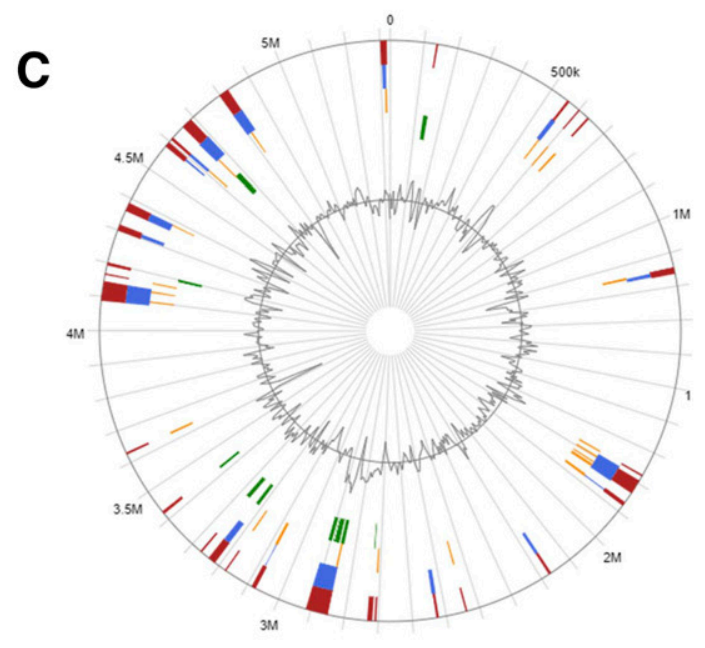

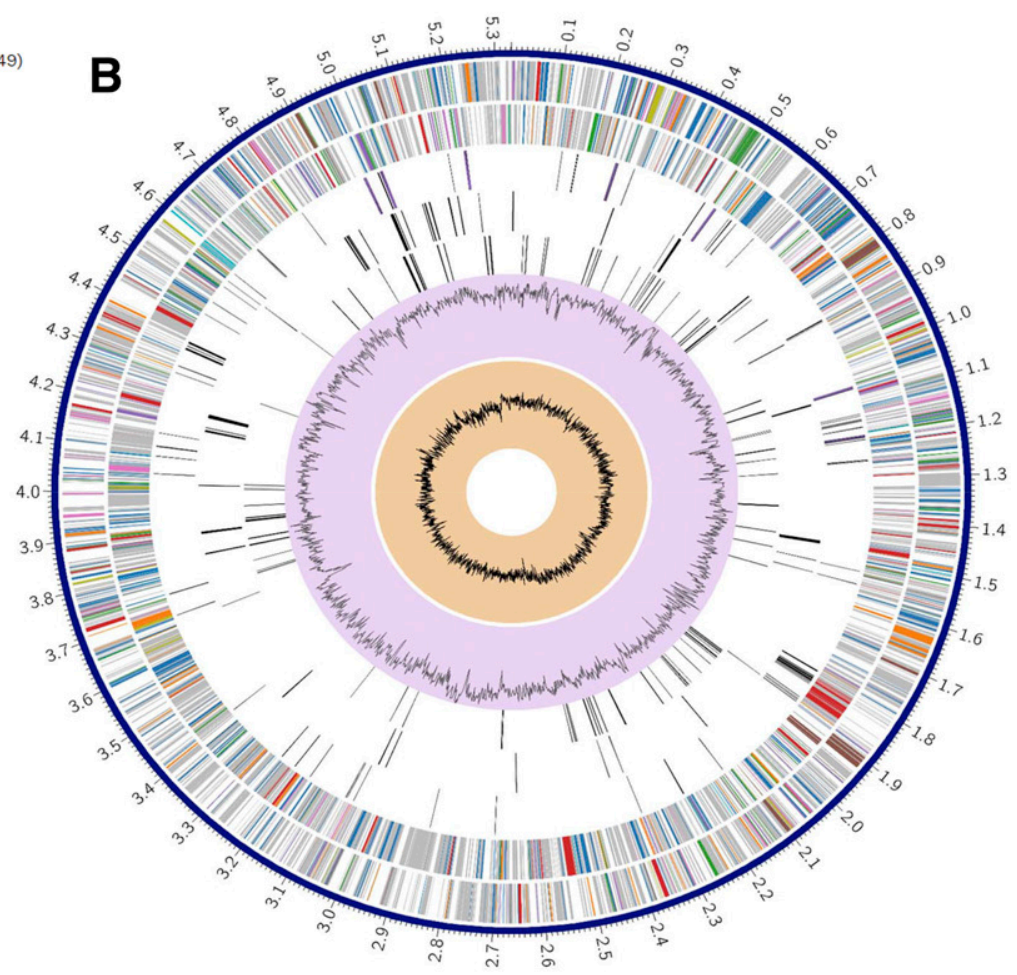

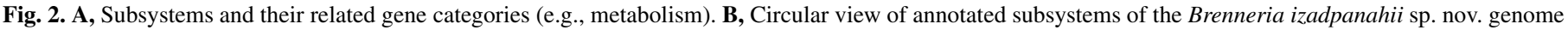
generated in PATRIC (Pathosystems Resource Integration Center). C, Pathogenicity islands screening using Island Viewer tool. 
A

$$
\begin{aligned}
& \text { Brenneria izadpanahii } \\
& \text { base singletons } \\
& \text { non-core } \\
& \text { core }
\end{aligned}
$$

Pangenome

anon-core

core
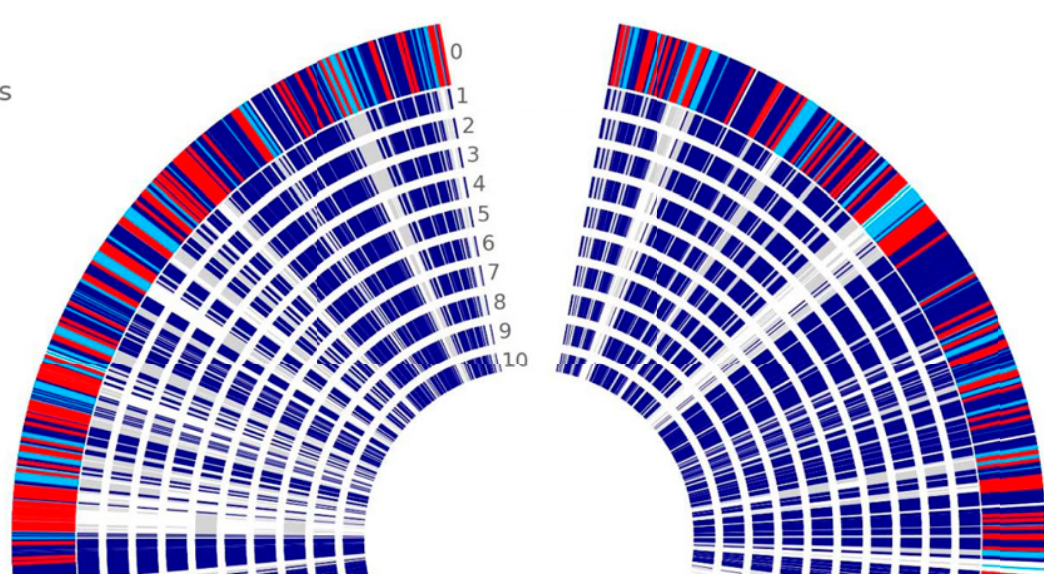

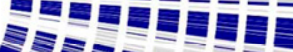

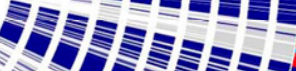

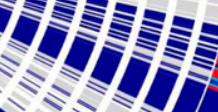

110101010

IIIIIII)

미 IIII)

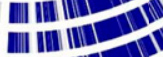

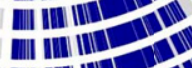

ail III IIIT

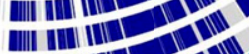

ain int ill

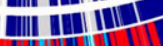

Pangenome A

Genome 0 Brenneria izadpanahii

Genome 1 Brenneria goodwinii 141

Genome 2 Brenneria goodwinii 171

Genome 3 Brenneria goodwinii OBR1

Genome 4 Brenneria corticis

Genome 5 Brenneria nigrifluens

Genome 6 Brenneria roseae ssp. americana

Genome 7 Brenneria roseae ssp. roseae

Genome 8 Brenneria alni

Genome 9 Brenneria salicis

Genome 10 Brenneria rubrifaciens
B

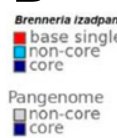

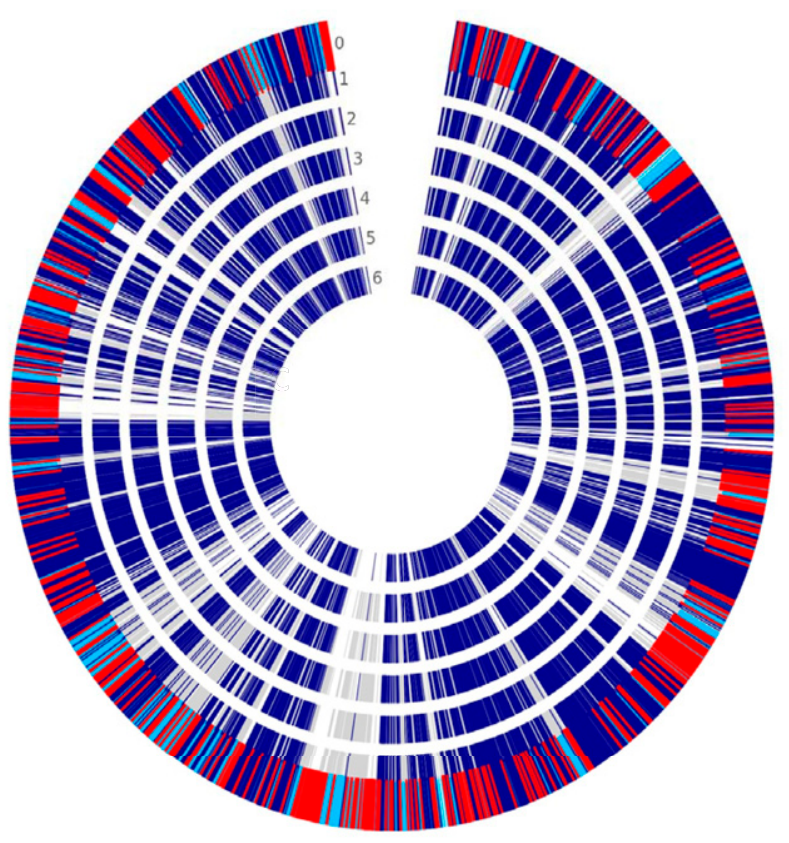

Genome 0 Brenneria izadpanahii Genome 1 Brenneria goodwinii 141

Genome 2 Brenneria goodwinii 171 Genome 3 Brenneria goodwinii OBR1

Genome 4 Brenneria corticis Genome 5 Brenneria nigrifluens

Genome 6 Brenneria alni

C

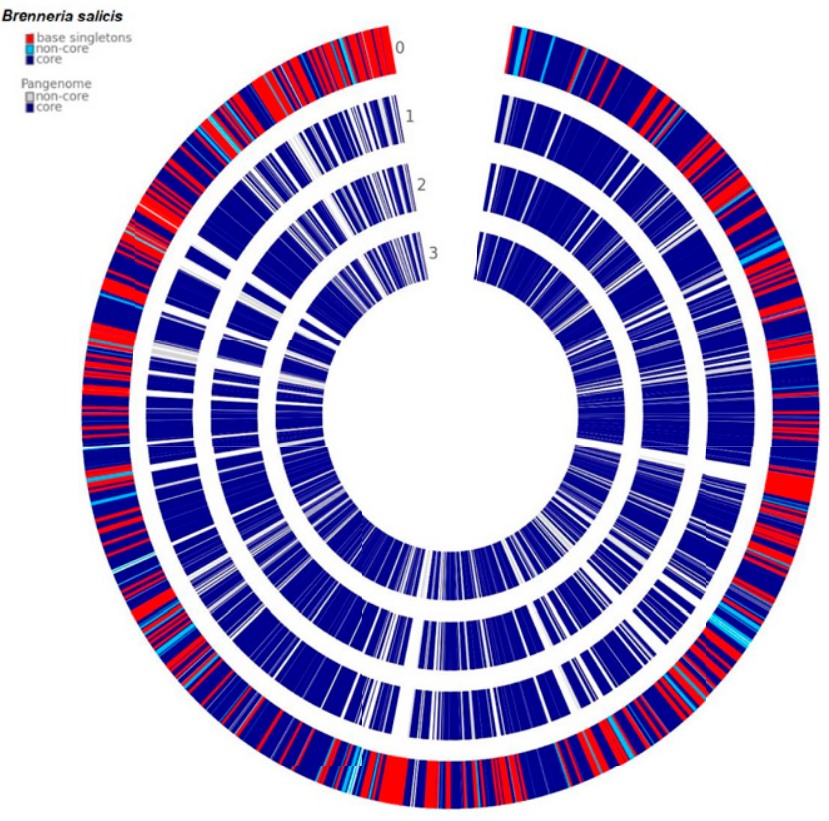

Genome 0 Brenneria salicis

Genome 1 Brenneria roseae ssp. roseae

Genome 2 Brenneria roseae ssp. americana

Genome 3 Brenneria rubrifaciens

Fig. 3. Pangenome analysis of Brenneria A, clade A+B species, B, clade A, and C, clade B performed using the Build Pangenome with OrthoMCL app (Pangenome Orthomcl version 0.0.7). Red (medium gray) indicates base singletons; and sky blue (light gray) represents noncore genes with respect to B. izadpanahii (A and B) and B. salicis (C) as based genomes compared with the respective pangenomes. Dark blue (dark gray) represents core genes. 
the most encoded metabolites/antibiotic factors within the genome dataset using antiSMASH database-bacterial version (Table 6).

\section{DISCUSSION}

Only a few species within the genus Brenneria have been described so far despite increasing reports demonstrating that strains of Brenneria spp. are ubiquitous and commonly associated with woody plants presenting symptoms including bark canker and stem bleeding as the most visible (Brady et al. 2014; Denman et al. 2012; Hauben et al. 1998; Li et al. 2015, 2019; Zheng et al. 2017). The relatively small number of species analyzed is a likely consequence of the media and culturing conditions used in laboratory procedures and associated isolation biases. Also, screening of the population structure and evolutionary potential in genomic scales could be a useful tool since these bacteria may share close genetic relationships (e.g., B. goodwinii in U.K. [Kaczmarek et al. 2017]). So, combination of genomic indices provides added resolution to effectively identify species within the genus.

A genome-based phylogeny using 50 clusters of orthologous groups (Fig. 1B) proposed rearrangement necessity of the family Pectobacteriaceae into two subfamilies, sharing most common recent ancestor in Brenneria-Pectobacterium and Dickeya-Lonsdalea, respectively. The B. izadpanahii-B. goodwinii 141, B. nigrifluens- $B$. corticis, and $B$. roseae ssp. roseae- $B$. roseae ssp. americana formed very tight pairs related by almost $90 \%$ for B. izadpanahii-B. goodwinii pair and 95 to $96 \%$ for another two pairs, respectively, with all ANI calculations and reciprocal relatedness of the $\mathrm{dDDH}$ values. These results are consistent with the 16S rRNA gene/MLSA sequence similarity data (Fig. 1A). All other comparisons between Brenneria clades A and B gave even

TABLE 3. Summary statistics of the pangenome datasets and shared genes within Brenneria clade A and B species retrieved from KBase

\begin{tabular}{|c|c|c|c|}
\hline Organism (accession) & Number of genes in homologs & Number of homolog families & Number of genes in singleton \\
\hline B. izadpanahii (Iran 50) (CP050854) & 4,208 & 4,060 & 535 \\
\hline B. goodwinii FRB141 (CP014137) & 4,423 & 4,293 & 201 \\
\hline B. goodwinii FRB171 (MJLY00000000) & 4,505 & 4,374 & 143 \\
\hline B. goodwinii OBR1 (CGIG00000000) & 4,506 & 4,377 & 197 \\
\hline B. nigrifluens ATCC 13028 (CP034036) & 4,032 & 3,918 & 360 \\
\hline B. corticis $\mathrm{gBX} 10-1-2(\mathrm{QDKH} 00000000)$ & 4,022 & 3,895 & 479 \\
\hline B. alni NCPPB 3934 (MJLZ00000000) & 3,386 & 3,312 & 428 \\
\hline B. salicis DSM30166 (MJMA00000000) & 3,246 & 3,170 & 376 \\
\hline B. rubrifaciens 6D370 (CP034035) & 3,210 & 3,082 & 311 \\
\hline $\begin{array}{l}\text { B. roseae ssp. americana LMG } 27715 \\
\text { (NZ_QDKJ00000000.1) }\end{array}$ & 3,871 & 3,780 & 540 \\
\hline $\begin{array}{l}\text { B. roseae ssp. roseae LMG } 27714 \\
\text { (QDKI00000000) }\end{array}$ & 3,882 & 3,767 & 471 \\
\hline
\end{tabular}
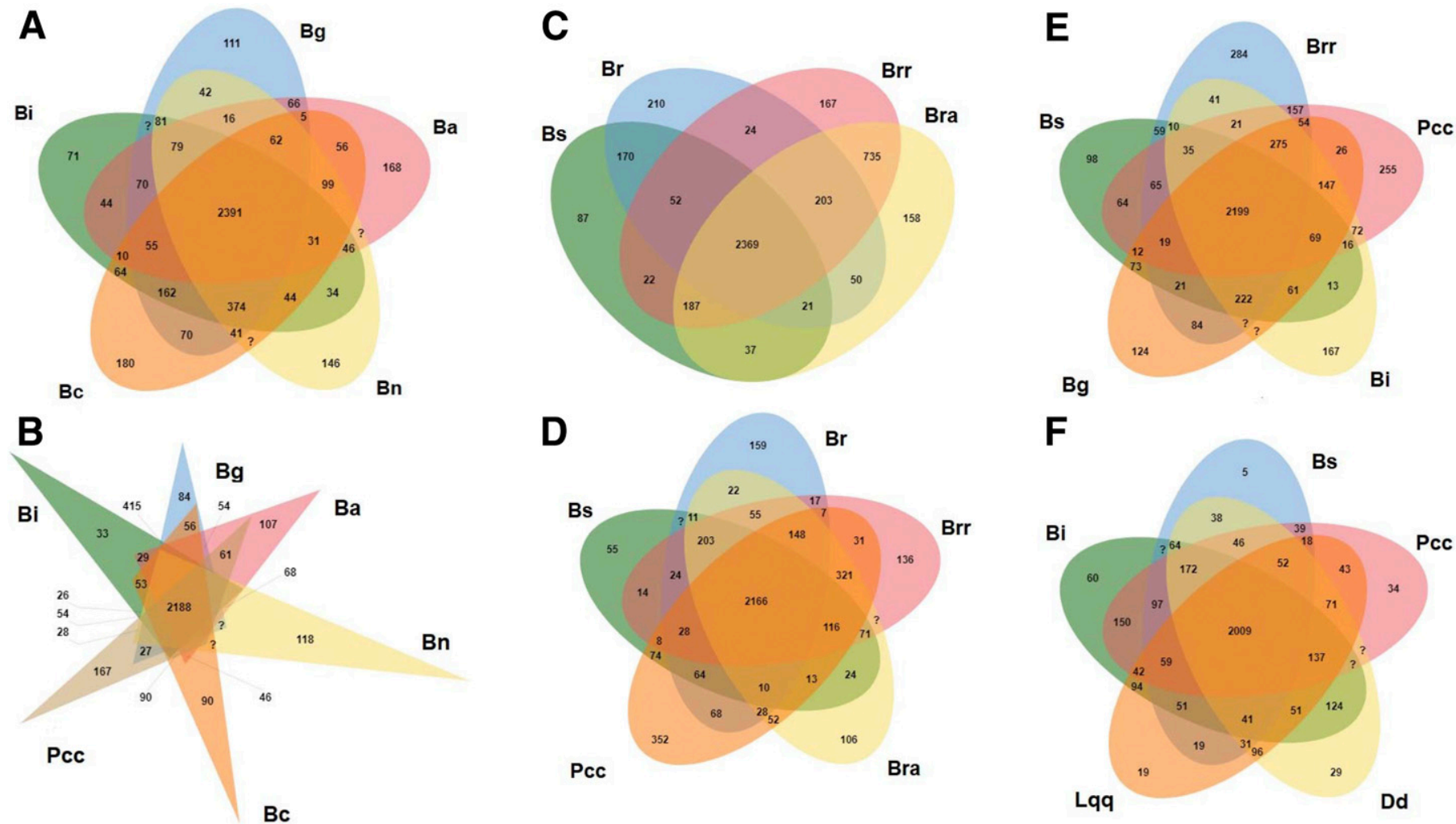



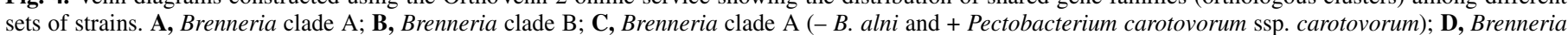

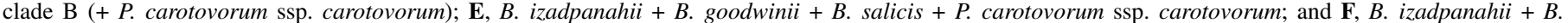
goodwinii + B. salicis + Lonsdaleae quercina ssp. quercina + P. carotovorum ssp. carotovorum + Dickeya dadantii . 
lower similarity levels (below-threshold genomic similarity) and were scattered in several clades. Thus, the novel isolate should also be considered as another new species from a genomic point of view (Fig. 5A).

With advances in high-throughput sequencing technologies bacterial taxonomy is undergoing many changes as a consequence and new innovative tools were developed (Caputo et al. 2019). Using pangenomic analyses, species can be redefined or new species definitions generated. The analysis pipeline in KBase was useful in providing a phylogenetic and functional genomic assessment of many whole bacterial genomes simultaneously. The Brenneria species were divided according to their host plants, the disease symptoms they cause, and other phenotypic or genotypic features. The taxonomic classification of Brenneria species has been the subject of controversy (Denman et al. 2012; Hauben et al. 1998). In a recent update, B. quercina was transferred to the new genus Lonsdalea (Brady et al. 2012). Here, we analyzed core/ noncore and singleton genes in pangenomes once for genome-based taxonomy point of view in addition to deciphering their common and special potential activities.

Both the core and dispensable genes are crucial for determining bacterial species diversity that are responsible for fundamental functions including replication, translation, and maintenance of cellular homeostasis and survivability, antimicrobial resistance, virulence traits, and development of novel gene functions, respectively (Adeniji et al. 2019).

A majority of the core genes irrespective of their functions and location in the genome are useful for phylogenomic reassignments among Brenneria and elsewhere. This pangenomic analysis enabled us to conclude that B. izadpanahii, which exhibit as many differences between them as with other Brenneria species, is a distinct Brenneria species. The conserved genomic regions of the Brenneria strains were probably due to their common ancestry, either by duplication or by speciation of the genes (Fig. 1C). While

A
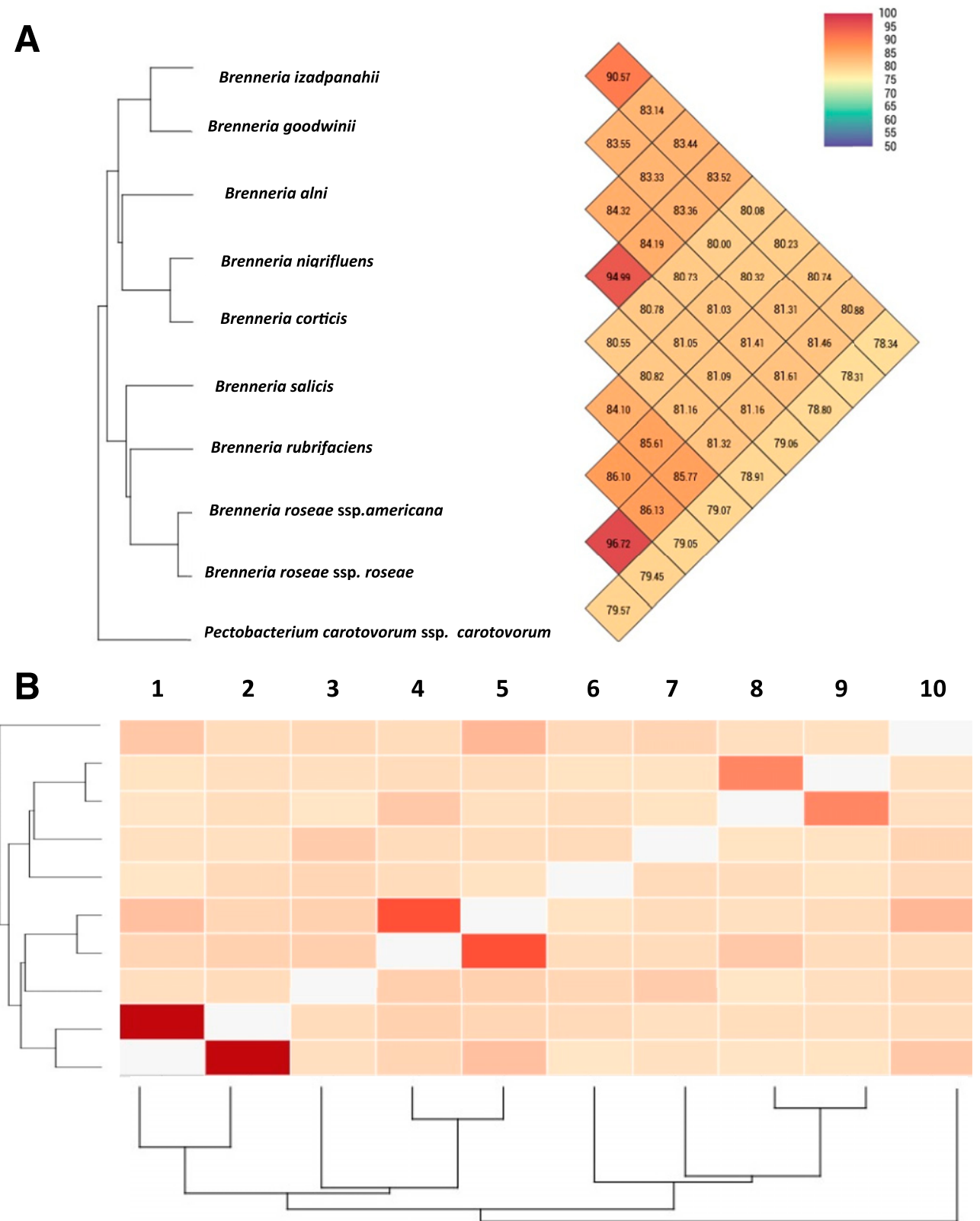

10-Pectobacterium carotovorum ssp. carotovorum 9-Brenneria roseae ssp. roseae 8-Brenneria roseae ssp.americana 7-Brenneria rubrifaciens 6-Brenneria salicis 5-Brenneria corticis 4-Brenneria niarifluens 3-Brenneria alni 2-Brenneria goodwinii 1- Brenneria izadpanahii

Fig. 5. A, Average nucleotide identity (ANI)-based neighbor joining phylogenetic tree of Brenneria clade A and B species in addition to Pectobacterium carotovorum ssp. carotovorum constructed using the OrthoANI calculator software. Different colors represent degree of identity. B, Heatmap showing intrastrain similarities of orthologous subsets from the Brenneria clade A and B species in addition to P. carotovorum ssp. carotovorum generated by the OrthoVenn 2 online service. 

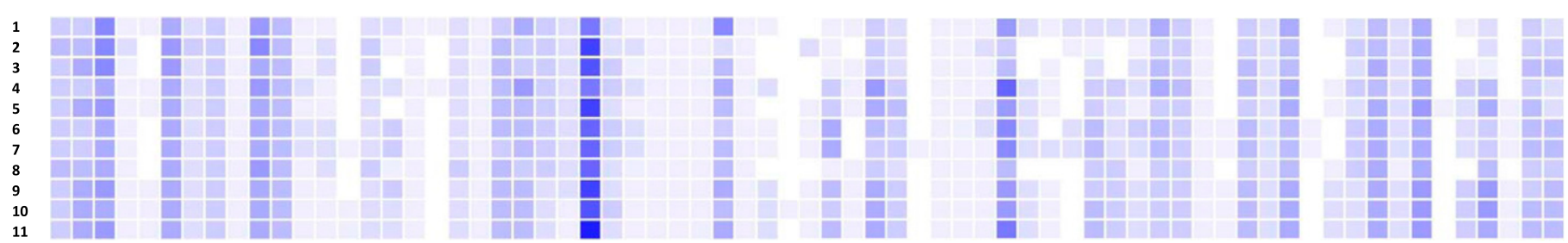

70

80

90

100

110

120

130
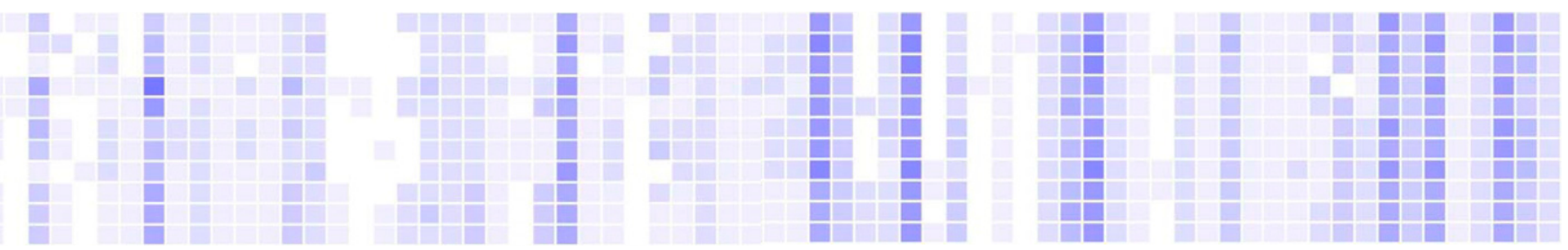

\section{0}

150

160

170

180

190



200
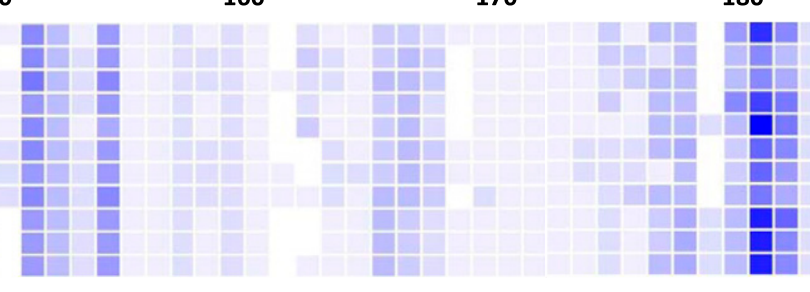

240

250

260

270

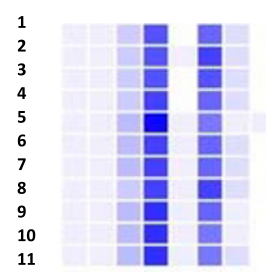

220
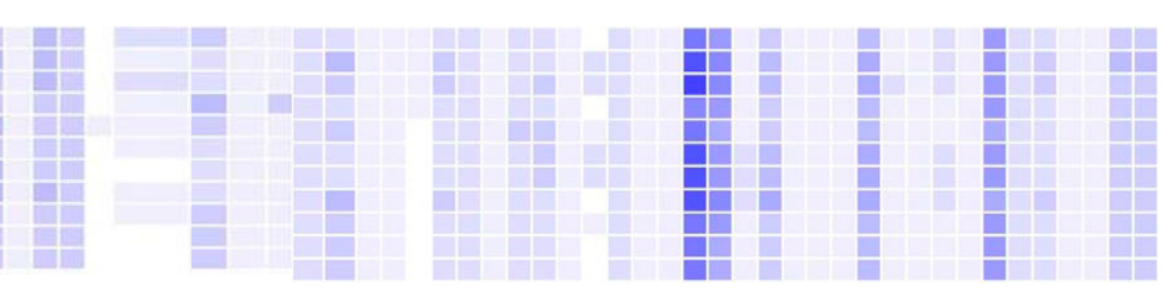

280

290

300

310

320

330

340
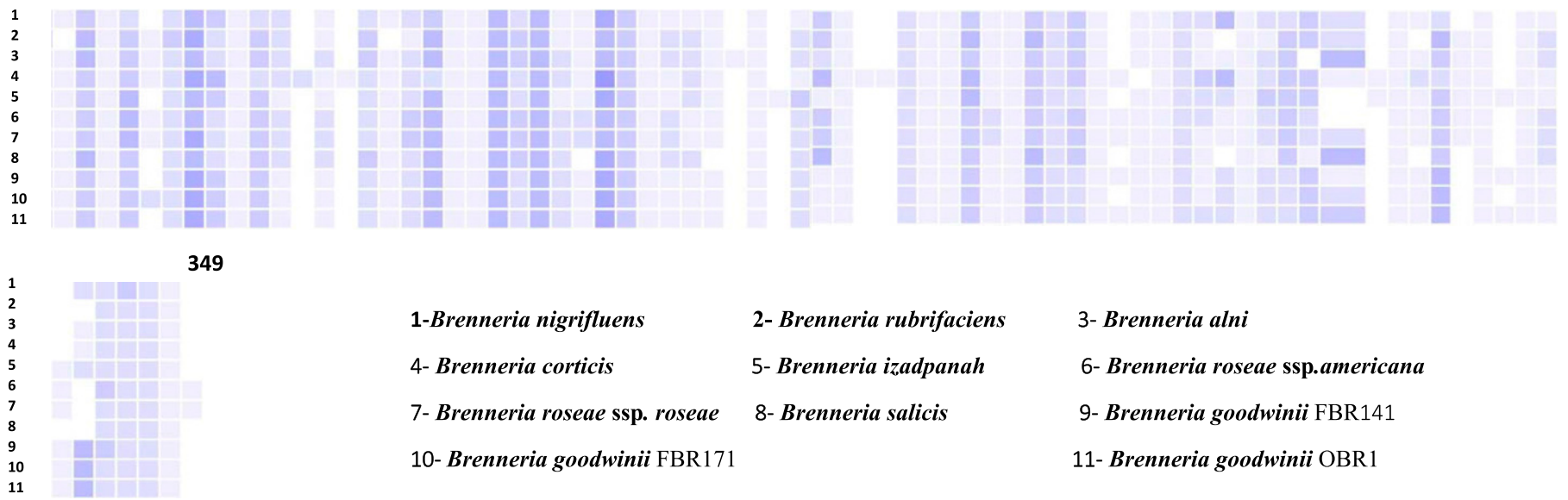

\section{1-Brenneria nigrifluens \\ 4- Brenneria corticis \\ 2- Brenneria rubrifaciens \\ 5- Brenneria izadpanah \\ 7- Brenneria roseae ssp. roseae \\ 8- Brenneria salicis \\ 10- Brenneria goodwinii FBR 171}

\section{3- Brenneria alni \\ 6- Brenneria roseae ssp.americana \\ 9- Brenneria goodwinii FBR141 \\ 11- Brenneria goodwinii OBR1}

Fig. 6. Pangenome functional domains annotation of the Brenneria clade A and B species identified in the phylogenomic analysis using the SEED database.

Figure 6 KEY

Amino acids and derivatives

1-Alanine biosynthesis

2-Arginine biosynthesis extended

3-Arginine deiminase pathway

4-Arginine and ornithine degradation

5-Aromatic amino acid degradation

6-Aromatic amino acid interconversions with aryl acids

7-Branched-chain amino acid biosynthesis

8-Chorismate: Intermediate for synthesis of PAPA antibiotics, PABA, anthranilate, 3-hydroxyanthranilate and more

9-Chorismate synthesis
10-Common pathway for synthesis of aromatic compounds (DAHP synthase to chorismate)

11-Cysteine biosynthesis

12-Glutamine, glutamate, aspartate, and asparagine biosynthesis

13-Glycine and serine utilization

14-Glycine cleavage system

15-HMG CoA synthesis

16-Histidine biosynthesis

17-Histidine degradation

18-Ketoisovalerate oxidoreductase

19-L-2-amino-thiazoline-4-carboxylic acid-L-cysteine conversion

20-Lysine biosynthesis DAP pathway
21-Lysine degradation

22-Methionine biosynthesis

23-Methionine degradation

24-Methionine salvage

25-Phenylalanine and tyrosine branches from chorismate

26-Polyamine metabolism

27-Proline, 4-hydroxyproline uptake and utilization

28-Proline synthesis

29-Serine biosynthesis

30-Threonine and homoserine biosynthesis

31-Threonine degradation

32-Urea decomposition

33-Valine degradation 
Fig. 6. Key (Continued from previous page)

34-2-Ketogluconate utilization

35-2-Methylcitrate to 2-methylaconitate metabolism cluster

36-Acetoin, butanediol metabolism

37-Acetyl-CoA fermentation to butyrate

38-Alpha-acetolactate operon

39-Beta-glucoside metabolism

40-Butanol biosynthesis

41- $\mathrm{CO}_{2}$ uptake, carboxysome

42-Calvin-Benson cycle

43-Carboxysome

44-Chitin and $\mathrm{N}$-acetylglucosamine utilization

45-D-Galacturonate and D-glucuronate utilization

46-D-Sorbitol (D-glucitol) and L-sorbose utilization

47-D-Tagatose and galactitol utilization

48-D-Allose utilization

49-D-Galactarate, D-glucarate, and D-Glycerate catabolism

50-D-Galactonate catabolism

51-D-Gluconate and ketogluconates metabolism

52-D-Ribose utilization

53-Deoxyribose and deoxynucleoside catabolism

54-Di-Inositol-phosphate biosynthesis

55-Dihydroxyacetone kinases

56-Entner-Doudoroff pathway

57-Ethanolamine utilization

58-Fermentations: Mixed acid

59-Formaldehyde assimilation: Ribulose monophosphate pathway

60-Fructooligosaccharides (FOS) and raffinose utilization

61-Fructose utilization

62-Glycerol and glycerol-3-phosphate uptake and utilization

63-Glycogen metabolism

64-Glycolysis and gluconeogenesis

65-Glycolysis and gluconeogenesis, including archaeal enzymes

66-Hexose phosphate uptake system

67-Inositol catabolism

68-Isobutyryl-CoA to propionyl-CoA module

69-L-Arabinose utilization

70-L-Ascorbate utilization (and related gene clusters)

71-L-Fucose utilization

72-L-Fucose utilization temp

73-Lactate utilization

74-Lacto-N-biose I and galacto-N-biose metabolic pathway

75-Lactose and galactose uptake and utilization

76-Lactose utilization

77-Maltose and maltodextrin utilization

78-Mannitol utilization

79-Mannose metabolism

80-Methylglyoxal metabolism

81-N-Acetyl-galactosamine and galactosamine utilization

82-One-carbon metabolism by tetrahydropterines

83-Pentose phosphate pathway

84-Photorespiration (oxidative $\mathrm{C} 2$ cycle)

85-Predicted carbohydrate hydrolases

86-Propanediol utilization

87-Propionate-CoA to succinate module

88-Pyruvate/ferredoxin oxidoreductase

89-Pyruvate alanine serine interconversions

90-Pyruvate metabolism I: anaplerotic reactions, PEP

91-Pyruvate metabolism II: acetyl-CoA, acetogenesis from pyruvate

92-Ribitol, xylitol, arabitol, mannitol, and sorbitol utilization

93-Serine-glyoxylate cycle

94-Sucrose utilization

95-TCA cycle

96-Trehalose biosynthesis

97-Trehalose uptake and utilization
98-Unknown sugar utilization (cluster

9-Xylose

Cell division and cell cycle

100-Bacterial cytoskeleton

101-MukBEF chromosome condensation

102-Two cell division clusters relating to chromosome partitioning

Cell wall and capsule

103-CMP-N-acetylneuraminate biosynthesis

104-Capsular heptose biosynthesis

105-Colanic acid biosynthesis

106-KDO2-lipid A biosynthesis

107-LOS core oligosaccharide biosynthesis

108-Lipid A-Ara4N pathway (polymyxin resistance)

109-Lipid A modifications

110-Peptidoglycan biosynthesis

111-Rhamnose containing glycans

112-Sialic acid metabolism

113-Teichuronic acid biosynthesis

114-UDP-N-acetylmuramate from fructose-6-phos-

phate biosynthesis

115-dTDP-rhamnose synthesis

116-Linker unit-arabinogalactan synthesis

117-Mycolic acid synthesis

Clustering-based subsystems

118-Bacterial cell division

119-CBSS-214092.1.peg. 3450

120-CBSS-262719.3.peg.410

121-CBSS-343509.6.peg.2644

122-CBSS-562.2.peg.5158 SK3 including

123-Cluster-based subsystem grouping hypotheticals-perhaps proteosome related

124-Conserved gene cluster associated with Met-

tRNA formyltransferase

125-LMPTP YwlE cluster

126-NusA-TFII Cluster

127-Putative hemin transporter

128-Putative sulfate assimilation cluster

129-Type III secretion systems, extended

130-YeiH

Cofactors, vitamins, prosthetic groups, pigments

131-Biotin biosynthesis

132-Coenzyme A biosynthesis

133-Coenzyme B12 biosynthesis

134-Experimental type

135-Flavodoxin

136-Folate biosynthesis

137-Heme and siroheme biosynthesis

138-Lipoic acid metabolism

139-Menaquinone and phylloquinone biosynthesis

140-Molybdenum cofactor biosynthesis

141-NAD and NADP cofactor biosynthesis global

142-NAD regulation

143-Plastoquinone biosynthesis

144-Pyridoxin (vitamin B6) biosynthesis

145-Pyrroloquinoline quinone biosynthesis

146-Riboflavin, FMN, and FAD metabolism

147-Thiamin biosynthesis

148-Ubiquinone biosynthesis

149-p-Aminobenzoyl-glutamate utilization

DNA metabolism

150-2-phosphoglycolate salvage

151-CRISPRs

152-DNA-replication

153-DNA repair base excision

154-DNA repair, UvrABC system

155-DNA repair, bacterial

156-DNA repair, bacterial DinG, and relatives

157-DNA repair, bacterial MutL-MutS system

158-DNA repair, bacterial RecFOR pathway

159-DNA repair, bacterial UvrD, and related helicases

160-DNA structural proteins, bacterial

161-DNA topoisomerases, Type I, ATPindependent
162-Plasmid replication

163-Restriction-modification system

164-RuvABC plus a hypothetical

165-YcfH

Fatty acids, lipids, and isoprenoids

166-Fatty acid biosynthesis FASII

167-Glycerolipid and glycerophospholipid metab-

olism in bacteria

168-Isoprenoid biosynthesis

169-Polyhydroxybutyrate metabolism

170-Triacylglycerol metabolism

171-Polyprenyl synthesis

Iron acquisition and metabolism

172-Campylobacter iron metabolism

173-Heme, hemin uptake and utilization systems in gram positives

174-Hemin transport system

175-Iron acquisition in Vibrio

176-Siderophore aerobactin

177-Siderophore enterobactin

178-Transport of iron

Membrane transport

179-ABC transporter alkylphosphonate (TC 3.A.1.9.1)

180-ABC transporter branched-chain amino acid

(TC 3.A.1.4.1)

181-ABC transporter dipeptide (TC 3.A.1.5.2)

182-ABC transporter oligopeptide (TC 3.A.1.5.1)

183-ABC transporter peptide (TC 3.A.1.5.5)

184-Fructose and mannose inducible PTS

$185-\mathrm{Na}(+) \mathrm{H}(+)$ antiporter

186-Ton and Tol transport systems

187-Transport of manganese

188-Transport of nickel and cobalt

189-Transport of zinc

190-Twin-arginine translocation system

191-Type 4 conjugative transfer system, IncI1 type

192-Type III secretion system orphans

193-Type IV pilus

194-Widespread colonization island

195-pVir Plasmid of Campylobacter

Metabolism of aromatic compounds

196-Aromatic amin catabolism

197-Benzoate degradation

198-Biphenyl degradation

199-Catechol branch of beta-ketoadipate pathway

200-Central meta-cleavage pathway of aromatic compound degradation

201-Homogentisate pathway of aromatic compound degradation

202-Phenylpropanoid compound degradation

203-Protocatechuate branch of beta-ketoadipate pathway

204-Quinate degradation

205-Salicylate and gentisate catabolism

Miscellaneous

206-Conserved gene cluster possibly involved in RNA metabolism

207-Muconate lactonizing enzyme family

208-YaaA

209-YbbK

210-ZZ gio need homes

Motility and chemotaxis

211-Bacterial chemotaxis

212-Flagellar motility

213-Flagellum

214-Flagellum in Campylobacter

Nitrogen metabolism

215-Allantoin utilization

216-Amidase clustered with urea and nitrile hydratase functions

217-Ammonia assimilation

218-Cyanate hydrolysis

219-Dissimilatory nitrite reductase

220-Nitrate and nitrite ammonification

221-Nitric oxide synthase

222-Nitrogen fixation 
the phylogenomic position of the Dickeya-Pectobacterium clade was clarified in these analyses, only further investigations using a larger collection of strains will shed light on the genetic content and taxonomic status of the family Pectobacteriaceae.

Although the existence of T3SS cluster within a putative pathogen is typically considered a substantial factor in establishing the causality of disease (Arnold and Jackson 2011), it should be noted that the functional hrp systems as a minor virulence component in the Pectobacteriaceae (Pectobacterium and Dickeya) (Pérombelon 2002) may reflect a hemibiotrophic lifestyle of Brenneria ssp. (Glazebrook 2005). While B. corticis with high similarity in genome metrics and functional domains with the $B$. nigrifluens (a well-known pathogenic member of the genus) does not carry the T3SS cluster and potential effectors, only further investigations could decipher exact nature of the bacterium in related pathosystem. The whole genome sequence analyses Lee et al. (2020) showed that the nonpathogenic Xanthomonas campestris strain $\mathrm{nE} 1$ does not contain the entire pathogenicity island ( $h r p$ gene cluster; type III secretion system) and all type III effector protein genes. Experimental evidence demonstrated that this nonpathogenic strain (nE1) could acquire the entire pathogenicity island from the endemic $X$. campestris pv. campestris and $X$. campestris pv. raphani strains by conjugation, while type III effector genes were not cotransferred (Lee et al. 2020). This results in raising questions in the case of symptomatic trees where several bacterial species occasionally are found as complex networks in the microbiome and the possible transfer events of pathogenicity determinants between the species is not impossible.

The analysis of prophage regions within Brenneria genomes may be fundamental to understanding their evolution and the potential for transfer for virulence factors between strains or species such as reported for ECA41 and hopAR1 in potato and cherry, respectively (Evans et al. 2010; Hulin et al. 2018), and influencing ecological fitness of their bacterial hosts (e.g., genes encoding metal ion transporters) (Matos et al. 2013; Ohnishi et al. 2001). Thirty-seven intact prophages have been characterized in Pectobacterium spp. and Dickeya spp. genomes and 6 of 37 were located near genes coding for pectate lyase degrading enzyme (Czajkowski 2019). The relatively high number of intact prophages found in the present analysis may suggest that the interaction of Brenneria and

Fig. 6. Key (Continued from previous page)

223-Nitrosative stress

Nucleosides and nucleotides

224-De novo purine biosynthesis

225-De novo pyrimidine synthesis

226-Hydantoin metabolism

227-Nudix proteins (nucleoside triphosphate hydrolases)

228-Purine utilization

229-Purine conversions

230-Purine nucleotide synthesis regulator

231-Pyrimidine utilization

232-Ribonucleotide reduction

233-Xanthosine utilization (xap region)

Phages, prophages, transposable elements, plasmids

234-Phage capsid proteins

235-Staphylococcal pathogenicity islands SaPI

236-Staphylococcal phi-Mu50B-like prophages

Phosphorus metabolism

237-Alkylphosphonate utilization

238-Phosphate metabolism

239-Phosphonate metabolism

Potassium metabolism

240-Glutathione-regulated potassium-efflux system and associated functions

241-Potassium homeostasis Protein metabolism

242-Glycine reductase, sarcosine reductase, and betaine reductase

243-GroEL GroES

244-N-linked glycosylation in bacteria

245-Peptidyl-prolyl cis-trans isomerase

246-Periplasmic disulfide interchange

247-Programmed frameshift

248-Proteasome bacterial

249-Protein chaperones

250-Protein degradation

251-Protein secretion by ABC-type exporters

252-Proteolysis in bacteria, ATP-dependent

253-Putative TldE-TldD proteolytic complex

254-Ribosomal protein S12p Asp methylthiotransferase

255-Ribosome LSU bacterial

256-Ribosome SSU bacterial

257-Ribosome activity modulation

258-Ribosome biogenesis bacterial

259-Translation elongation factor $G$ family

260-Translation elongation factors eukaryotic and archaeal

261-Translation initiation factors eukaryotic and archaeal

262-Universal GTPases

263-tRNA aminoacylation, Asp, and Asn

264-tRNA aminoacylation, Glu, and Gln
RNA metabolism

265-ATP-dependent RNA helicases, bacterial

266-Polyadenylation bacterial

267-Queuosine-archaeosine biosynthesis

268-RNA polymerase bacterial

269-RNA processing and degradation, bacterial

270-Ribonuclease $\mathrm{H}$

271-Rrf2 family transcriptional regulators

272-Transcription factors bacterial

273-Transcription initiation, bacterial sigma factors

274-Wyeosine-MimG biosynthesis

275-tRNA processing

Regulation and cell signaling

276-CytR regulation

277-DNA-binding regulatory proteins, strays

278-MazEF toxin-antitoxin (programmed cell death) system

280-Murein hydrolase regulation and cell death

279-Orphan regulatory proteins

280-Sex pheromones in Enterococcus faecalis and other firmicutes

281-Stringent response, (p)ppGpp metabolism

282-cAMP signaling in bacteria

Respiration

283-Anaerobic respiratory reductases

284-Biogenesis of c-type cytochromes

285-Biogenesis of cytochrome c oxidases

286-Carbon monoxide induced hydrogenase

287-F0F1-type ATP synthase

288-Flavocytochrome C

289-Formate dehydrogenase

290-Formate hydrogenase

291-Hydrogenases

292-Membrane-bound Ni, Fe-hydrogenase

293-Na(+)-translocating NADH-quinone

294-NiFe hydrogenase maturation

295-Respiratory complex I

296-Respiratory dehydrogenases 1

297-Soluble cytochromes and functionally related electron carriers

298-Succinate dehydrogenase

299-Terminal cytochrome oxidases

300-Trimethylamine N-oxide (TMAO)

reductase

Secondary metabolism

301-Auxin biosynthesis

302-Biflavanoid biosynthesis

303-Cinnamic acid degradation

304-Phenazine biosynthesis

305-Steroid sulfates

306-Tannin biosynthesis
Stress response

307-Bacterial hemoglobins

308-Choline and betaine uptake and betaine biosynthesis

309-D-trosyl-tRNA(Tyr) deacylase

310-Ectoine biosynthesis and regulation

311-Flavohemoglobin

312-Glutaredoxins

313-Glutathione-dependent pathway of formaldehyde detoxification

314-Glutathione: Biosynthesis and gamma-glutamyl cycle

315-Glutathione: Nonredox reactions

316-Glutathione: Redox cycle

317-Glutathionylspermidine and trypanothione

318-Heat shock dnaK gene cluster extended

319-Hfl operon

320-Oxidative stress

321-Redox-dependent regulation of nucleus processes

322-Rubrerythrin

323-SigmaB stress response regulation

324-Sugar-phosphate stress regulation

325-Universal stress protein family

Sulfur metabolism

326-Alkanesulfonate assimilation

327-Alkanesulfonates utilization

328-Sulfate reduction-associated complexes

329-Taurine utilization

330-Thioredoxin-disulfide reductase

331-Utilization of glutathione as a sulfur source

Virulence, disease, and defense

332-Type 4 secretion and conjugative transfer

333-Aminoglycoside adenylyltransferases

334-Arsenic resistance

335-Bacteriocin-like peptides Blp

336-Beta-lactamase

337-Cobalt-zinc-cadmium resistance

338-Copper homeostasis

339-Copper homeostasis: Copper tolerance

340-Fosfomycin resistance

341-MLST

342-Methicillin resistance in staphylococci

343-Multidrug resistance, 2-protein version found in gram-positive bacteria

344-Multidrug resistance, tripartite systems found in gram-negative bacteria

345-Multidrug resistance efflux pumps

346-Multidrug efflux pump in Campylobacter jejuni (CmeABC operon)

347-Resistance to fluoroquinolones

348-Streptococcus pyogenes recombinatorial zone

349-Zinc resistance 
(bacterio) phages at the microbiome scale is underestimated. The potential effects of phages in AOD is undescribed but is suggested by the upregulation of bacteriophages genes in the AOD pathobiome in which $B$. goodwinii was the dominant taxa and was an essential virulence component.

The factors that determine the interactions within phyllosphere microbial communities are poorly understood whether they are between different bacteria or between bacteria and other microorganisms. The production of microbial secondary metabolites and antibiotics might be the principal mechanisms by which endogenous bacteria and fungi antagonize each other (Vorholt 2012).

Aryl polyene are yellow pigments produced by bacteria living in widely varied environments such as soil, human intestines, or other ecological niches. Embedded in the membrane of the bacteria, they protect against oxidative stress or reactive oxygen species. (Grammbitter et al. 2019). The forces of biochemical adaptive evolution operate at the level of genes, manifesting in complex phenotypes and the global biodiversity of proteins and metabolites. Secondary metabolites characterizations aiming to decipher how these factors shape adaptive landscapes is an interesting era of bacterial plant pathogen studies. To our knowledge, the first evidence of derived type II polyketide synthases bacterial pigment across the family Pectobacteriaceae was documented in our in silico analysis.

The majority of detectable metabolites within the Brenneria genomes were related to the iron acquisition (Table 6). In response to iron limitation, many bacteria and some fungi produce siderophores. Turnerbactin is a triscatecholate siderophore that was first described in Teredinibacter turnerae, a shipworm endosymbiont found in decaying wood floating on water. This compound is crucial in obtaining iron, bacterial competition, and to the survival of the symbiont in nature (Han et al. 2013). The number of gene clusters related to iron acquisition and transfer within the Brenneria clades members (of various types) suggests they may be crucial to the successful colonization of their plant host. The in silico evidence presented here suggest that these factors could be important in epiphytic maintenance of bacterial species and that experimental evidence should be sought to discover their role in planta.

Our findings provide the first genome/pangenome-based approach conducted on the genus Brenneria and allowed the classification of our new isolate into novel species, whose description is given below. The genetic variations cataloged here provide new insights into the evolutionary history of the genus, generating hypotheses about phylogenetic relationships, common and new pathogenicity scenarios, and how possibly they may synchronize their functions as a dominant complex community of the Brenneria. Functionally distinctive domains (in singletons category) within the Brenneria species genomes that specifically massive enzymatic potential activity, transcriptional regulatory, and ABC transporters in many types encoded by $B$. izadpanahii and huge number of phage-related genes in B. goodwinii are only small examples that show the diversification and adaptive capabilities in the biology of Brenneria, and each species may act as a puzzle pieces. Other members encompass a variety batch of pathogenicity repertoires that enable them to settle an offensive front line against the host defense barriers and become dominant taxa in the microbiome. These data are consistent with our observations in the Iranian diseased oak pathosystem that isolation of multiple Brenneria

TABLE 4. The carbohydrate-active enzymes database (CAZym) top 10 most frequent and hit repertoires of the Brenneria species and their relevant family Pectobacteriaceae $^{\text {a }}$

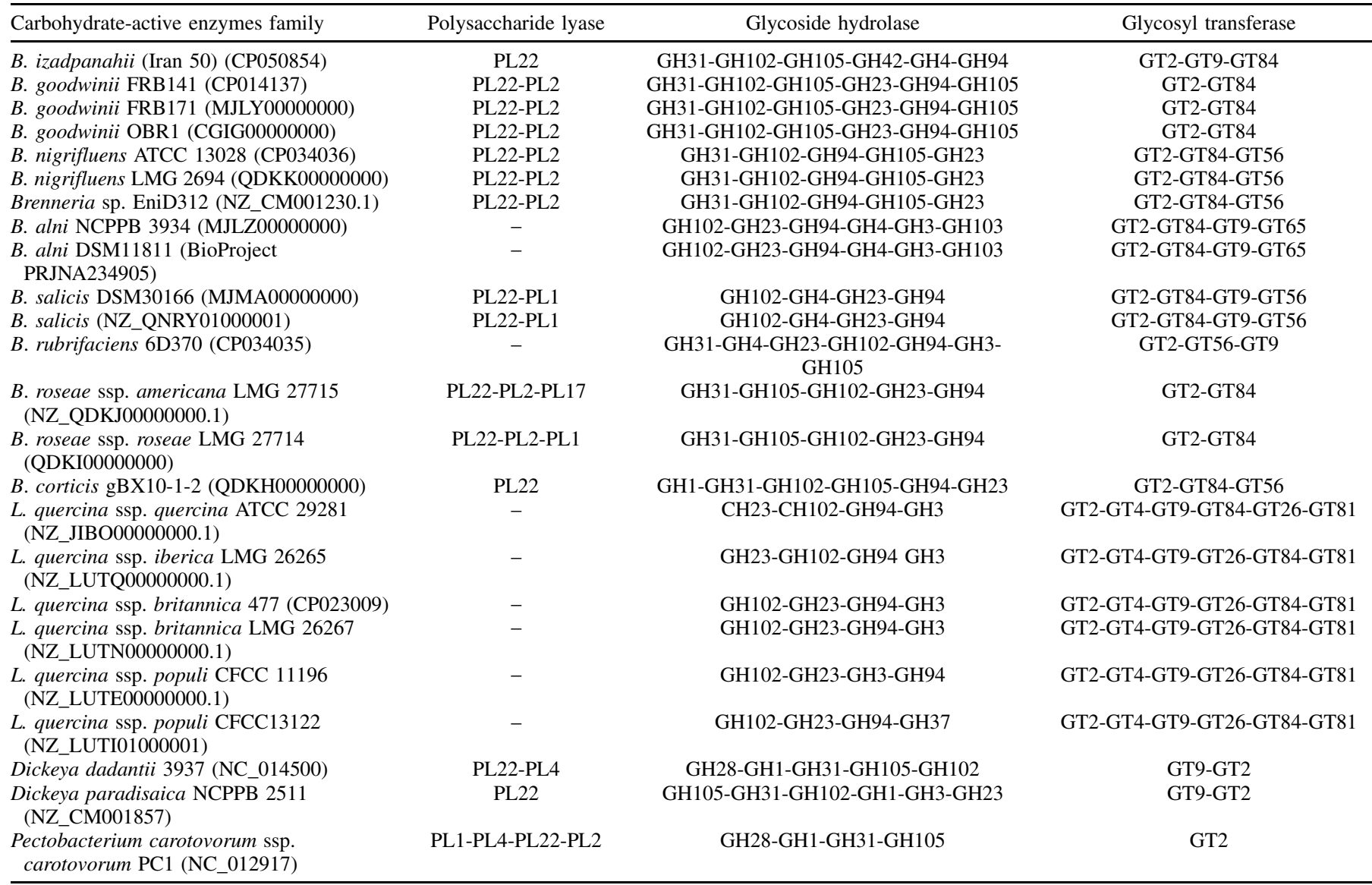

a The family carbohydrate esterase (CE) only had three most frequent and top hits for CE1 in Dickeya dadantii, D. paradisaica, and P. carotovorum ssp. carotovorum PC1. The family carbohydrate-binding (CB) module had no most frequent and top hit within the genomes investigated in this study (PL, polysaccharide lyase family; GH, glycoside hydrolases family; and GT, glycosyl transferase family). 
TABLE 5. Prophages within the genome sequences of Brenneria spp. and relevant Pectobacteriaceae strains detected using the online service PHASTER

\begin{tabular}{|c|c|c|c|c|c|c|}
\hline Organism (accession) & $\begin{array}{c}\text { Region } \\
\text { length }(\mathrm{kb})\end{array}$ & Completeness & $\begin{array}{c}\text { Number of } \\
\text { proteins }\end{array}$ & Position & Phage (GenBank accession number) & $\mathrm{GC} \%$ \\
\hline \multirow{3}{*}{$\begin{array}{l}\text { B. izadpanahii (Iran 50) } \\
(\mathrm{CP} 050854)^{* *}\end{array}$} & $10.5 \mathrm{~kb}$ & Incomplete & 14 & $2164668-2172805$ & PHAGE_Salmon_SEN1_NC_029003(3) & $45.56 \%$ \\
\hline & $32.6 \mathrm{~kb}$ & Intact & 37 & $4580442-4613088$ & PHAGE_Aeromo_phiO18P_NC_009542(16) & $49.60 \%$ \\
\hline & $17.7 \mathrm{~kb}$ & Incomplete & 10 & $5308310-5326077$ & PHAGE_Gordon_Schwabeltier_NC_031255(1) & $55.40 \%$ \\
\hline \multirow{3}{*}{$\begin{array}{l}\text { B. goodwinii FRB141 } \\
(\mathrm{CP} 014137)^{* *}\end{array}$} & $31.8 \mathrm{~kb}$ & Intact & 46 & $5067377-5099204$ & PHAGE_Vibrio_12B12_NC_021070(27) & $52.22 \%$ \\
\hline & $47.7 \mathrm{~kb}$ & Intact & 61 & $1117565-1165336$ & PHAGE_Entero_mEp237_NC_019704(8) & $49.35 \%$ \\
\hline & $47.2 \mathrm{~kb}$ & Intact & 50 & $5313441-5360697$ & PHAGE_Shigel_SfII_NC_021857(8) & $49.89 \%$ \\
\hline \multirow{3}{*}{$\begin{array}{l}\text { B. goodwinii FRB171 } \\
(\mathrm{MJLY} 00000000)^{*}\end{array}$} & $37.5 \mathrm{~kb}$ & Intact & 51 & $100426-137944$ & PHAGE_Pectob_ZF40_NC_019522(20) & $49.01 \%$ \\
\hline & $26.9 \mathrm{~kb}$ & Questionable & 15 & $254-27201$ & PHAGE_Salini_M1EM_1_NC_042348(1) & $46.24 \%$ \\
\hline & $12.7 \mathrm{~kb}$ & Incomplete & 9 & $56752-69526$ & PHAGE_Paenib_Fern_Ne-NC_028851(1) & $49.67 \%$ \\
\hline $\begin{array}{l}\text { B. goodwinii OBR1 } \\
(\text { CGIG00000000)** }\end{array}$ & $34.6 \mathrm{~kb}$ & Intact & 45 & $4675256-4709932$ & PHAGE_Pectob_ZF40_NC_019522(18) & $49.21 \%$ \\
\hline \multirow{9}{*}{$\begin{array}{l}\text { B. nigrifluens ATCC } \\
13028(\mathrm{CP} 034036)^{* *}\end{array}$} & $35.7 \mathrm{~kb}$ & Intact & 43 & 2091919-2127630 & PHAGE_Salmon_118970_sal3_NC_031940(11) & $52.93 \%$ \\
\hline & $34.4 \mathrm{~kb}$ & Intact & 46 & $2352275-2386708$ & PHAGE_Mannhe_vB_MhM_3927ĀP2_NC_028766(15) & $52.77 \%$ \\
\hline & $48.9 \mathrm{~kb}$ & Intact & 52 & $2587333-2636302$ & PHAGE_Pectob_ZF40_NC_019522(32) & $52.43 \%$ \\
\hline & $46.1 \mathrm{~kb}$ & Intact & 58 & 2909769-2955959 & PHAGE_Pectob_ZF40_NC_019522(18) & $50.91 \%$ \\
\hline & $35.2 \mathrm{~kb}$ & Intact & 48 & $3485094-3520326$ & PHAGE_Burkho_B̄eepMu_NC_005882(34) & $54.95 \%$ \\
\hline & $34.6 \mathrm{~kb}$ & Intact & 46 & $4625062-4659760$ & PHAGE_Salmon_SEN5_NC_028701(22) & $51.13 \%$ \\
\hline & $14.8 \mathrm{~kb}$ & Incomplete & 17 & $1439155-1453976$ & PHAGE_Entero_P4_NC_001609(3) & $49.87 \%$ \\
\hline & $33.4 \mathrm{~kb}$ & Incomplete & 13 & $2066903-2100388$ & PHAGE_Salmon_118970_sal3_NC_031940(3) & $50.32 \%$ \\
\hline & $27.8 \mathrm{~kb}$ & Incomplete & 14 & $4155884-4183780$ & PHAGE_Ācinet_vB_EAbaS_TRS1_NC_031098(1) & $49.08 \%$ \\
\hline \multirow{9}{*}{$\begin{array}{l}\text { Brenneria sp. EniD312 } \\
(\text { NZ_CM001230.1)** }\end{array}$} & $14.8 \mathrm{~kb}$ & Incomplete & 17 & $1433989-1448810$ & PHAGE_Entero_P4_NC_001609(3) & $49.87 \%$ \\
\hline & $62.5 \mathrm{~kb}$ & Intact & 61 & $2065618-2128139$ & PHAGE_Salmon_118970_sal3_NC_031940(14) & $52.29 \%$ \\
\hline & $34.4 \mathrm{~kb}$ & Intact & 45 & $2346736-2381169$ & PHAGE_Mannhe_vB_MhM_3927AP2_NC_028766(15) & $52.77 \%$ \\
\hline & $48.9 \mathrm{~kb}$ & Intact & 51 & $2581794-2630763$ & PHAGE_Pectob_ZF40_NC_019522(32) & $52.43 \%$ \\
\hline & $61.2 \mathrm{~kb}$ & Intact & 58 & $2904230-2965489$ & PHAGE_Pectob_ZF40_NC_019522(18) & $50.83 \%$ \\
\hline & $35.2 \mathrm{~kb}$ & Intact & 48 & $3537380-3572612$ & PHAGE_Burkho_BcepMu_NC_005882(34) & $54.95 \%$ \\
\hline & $27.8 \mathrm{~kb}$ & Incomplete & 14 & $4208171-4236067$ & PHAGE_Acinet_vB_AbaS_TRS1_NC_031098(1) & $49.08 \%$ \\
\hline & $34.6 \mathrm{~kb}$ & Intact & 44 & $4677347-4712045$ & PHĀGE_Salmon_SEN5_NC_028701(22) & $53.13 \%$ \\
\hline & $8.9 \mathrm{~kb}$ & Incomplete & 11 & $4225894-4234795$ & PHAGE_Paenib_Fern_NC_028851(1) & $48.76 \%$ \\
\hline \multirow{5}{*}{$\begin{array}{l}\text { B. alni NCPPB } 3934 \\
(\mathrm{MJLZ00000000)**}\end{array}$} & $35.9 \mathrm{~kb}$ & Intact & 52 & $49840-85804$ & PHAGE_Vibrio_12B12_NC_021070(29) & $51.29 \%$ \\
\hline & $61.5 \mathrm{~kb}$ & Intact & 68 & $13957-75481$ & PHAGE_Escher_PA28_NC_041935(14) & $50.72 \%$ \\
\hline & $26.2 \mathrm{~kb}$ & Incomplete & 36 & $59809-86095$ & PHAGE_Burkho_BcepMu_NC_005882(22) & $54.45 \%$ \\
\hline & $64.5 \mathrm{~kb}$ & Intact & 83 & $28-64615$ & PHAGE_Pseudo_B3_NC_006548(20) & $54.90 \%$ \\
\hline & $15.9 \mathrm{~kb}$ & Incomplete & 21 & $2479-18388$ & PHAGE_Entero_vB_KleM_RaǨ2_NC_019526(1) & $43.98 \%$ \\
\hline \multirow{2}{*}{$\begin{array}{l}\text { B. salicis DSM30166 } \\
\text { (MJMA00000000)* }^{\text {MJMA }}\end{array}$} & $34.9 \mathrm{~kb}$ & Intact & 49 & $80150-115135$ & PHĀGE_Vibrio_12B12_NC_021070(29) & $51.00 \%$ \\
\hline & $11.6 \mathrm{~kb}$ & Intact & 17 & $209-11865$ & PHAGE_Escher_HK75_NC_016160(7) & $49.92 \%$ \\
\hline \multirow{4}{*}{$\begin{array}{l}\text { B. rubrifaciens 6D } 370 \\
(\mathrm{CP} 034035)^{* *}\end{array}$} & $33.5 \mathrm{~kb}$ & Intact & 41 & $3759662-3793182$ & PHAGE_Salmon_SEN5_NC_028701(20) & $51.50 \%$ \\
\hline & $21.8 \mathrm{~kb}$ & Incomplete & 9 & 2779933-2801797 & PHAGE_Escher_phiV10_NC_007804(2) & $51.55 \%$ \\
\hline & $18.6 \mathrm{~kb}$ & Questionable & 21 & $404008-422613$ & PHAGE_Entero_P4_NC_001609(1) & $51.30 \%$ \\
\hline & $23.5 \mathrm{~kb}$ & Questionable & 22 & $1352642-1376210$ & PHAGE_Paenib_Tripp_NC__028930(2) & $50.38 \%$ \\
\hline \multirow{4}{*}{$\begin{array}{l}\text { B. roseae ssp. americana } \\
\text { LMG } 27715 \\
(\mathrm{NZ} Q \mathrm{QDKJ} 00000000.1)^{*}\end{array}$} & $44.6 \mathrm{~kb}$ & Intact & 64 & 99117-143778 & PHAGE_Pectob_ZF40_NC_019522(28) & $50.48 \%$ \\
\hline & $37.0 \mathrm{~kb}$ & Intact & 34 & $111756-148839$ & PHAGE_Salmon_SEN5_NC_028701(15) & $53.48 \%$ \\
\hline & $28.9 \mathrm{~kb}$ & Incomplete & 17 & 294796-323702 & PHAGE_Pectob_ZF40_NC_019522(3) & $52.02 \%$ \\
\hline & $5.0 \mathrm{~kb}$ & Incomplete & 10 & $182444-187482$ & PHAGE_Sodali_phiSG1_NC_007902(2) & $45.47 \%$ \\
\hline \multirow{4}{*}{$\begin{array}{l}\text { B. roseae ssp. roseae } \\
\text { LMG } 27714 \\
(\mathrm{QDKI} 00000000)^{*} \\
\text { B. corticis } \mathrm{gBX} 10-1-2\end{array}$} & $23.4 \mathrm{~kb}$ & Incomplete & 15 & $49239-72733$ & PHAGE_Entero_mEp234_NC_019715(2) & $48.40 \%$ \\
\hline & $29.2 \mathrm{~kb}$ & Intact & 32 & $1-29251$ & PHAGE_Aeromo_phiO18P_NC_009542(14) & $49.85 \%$ \\
\hline & $14.8 \mathrm{~kb}$ & Intact & 17 & $101137-116026$ & PHAGE_Escher_pro483_NC_028943(9) & $52.78 \%$ \\
\hline & $30.3 \mathrm{~kb}$ & Incomplete & 37 & $1-30367$ & PHAGE_Escher_PA28_NC_041935(9) & $54.11 \%$ \\
\hline L. quercina ssp. quercina & $27.7 \mathrm{~kb}$ & Questionable & 44 & $99899-127641$ & PHAGE_Burkho_phiE255_NC_009237(20) & $59.37 \%$ \\
\hline ATCC 29281 & $15.4 \mathrm{~kb}$ & Questionable & 20 & $142035-157468$ & PHAGE_E_Escher_D108_NC_-̄o13594(2) & $50.12 \%$ \\
\hline$\left(\mathrm{NZ} \_J I B O 00000000.1\right)^{*}$ & $30.4 \mathrm{~kb}$ & Incomplete & 10 & $65883-96379$ & PHAGE_Bacill_Shanette_NC__028983(2) & $50.17 \%$ \\
\hline L. quercina ssp. iberica & $12.1 \mathrm{~kb}$ & Incomplete & 16 & $111-12306$ & PHAGE_Burkho_BcepMu_NC_005882(13) & $61.31 \%$ \\
\hline $\begin{array}{l}\text { LMG } 26265 \\
\left(\mathrm{NZ} \_L U T Q 00000000\right)^{*}\end{array}$ & $5.3 \mathrm{~kb}$ & Incomplete & 9 & $132-5481$ & PHAGE_Salmon_SEN1_NC_029003(4) & $47.51 \%$ \\
\hline L. quercina ssp. & $36.2 \mathrm{~kb}$ & Intact & 49 & $42746-78945$ & PHAGE_Burkho_phiE255_NC_009237(22) & $58.53 \%$ \\
\hline britannica & $17.6 \mathrm{~kb}$ & Incomplete & 15 & $256669-274319$ & PHAGE_Entero_P4_NC_001609(2) & $50.41 \%$ \\
\hline $477(\mathrm{CP} 023009)^{* *}$ & $10.8 \mathrm{~kb}$ & Incomplete & 11 & $1666900-1677782$ & PHAGE_Salisa_1_NC_017983(1) & $51.15 \%$ \\
\hline & $52.4 \mathrm{~kb}$ & Intact & 66 & $2839465-2891943$ & PHAGE_Cronob_ENT47670_NC_019927(11) & $50.66 \%$ \\
\hline & $29.3 \mathrm{~kb}$ & Intact & 42 & $3985464-4014799$ & PHAGE_Burkho_BcepMu_NC_005882(20) & $59.88 \%$ \\
\hline L. quercina ssp. populi & $11.3 \mathrm{~kb}$ & Incomplete & 13 & $47358-58710$ & PHAGE_Escher_D108_NC_013594(2) & $51.58 \%$ \\
\hline CFCC 11196 & $10.3 \mathrm{~kb}$ & Incomplete & 18 & $3459-13797$ & PHAGE_Burkho_phiE255_NC_009237(9) & $56.03 \%$ \\
\hline$\left(\mathrm{NZ} \_L U T E 00000000.1\right)^{*}$ & $21.0 \mathrm{~kb}$ & Intact & 31 & $683-21688$ & PHAGE_Salmon_Fels_2_NC_010463(22) & $56.02 \%$ \\
\hline Dickèa dadantii 3937 & $32.8 \mathrm{~kb}$ & Intact & 44 & $915372-948193$ & PHAGE_Haemop_HP1_NC_001697(17) & $52.11 \%$ \\
\hline$\left(\mathrm{NC} \_014500\right)^{* *}$ & $6.9 \mathrm{~kb}$ & Incomplete & 10 & $1903506-1910478$ & PHAGE_Erwini_vB_EamM_RisingSun_NC_042018(2) & $50.87 \%$ \\
\hline & $23.6 \mathrm{~kb}$ & Questionable & 19 & $2024025-2047705$ & PHAGE_Entero_P4_NC_001609(3) & $50.12 \%$ \\
\hline & $27.8 \mathrm{~kb}$ & Intact & 33 & $2733074-2760902$ & PHAGE_Escher_pro483_NC_028943(12) & $56.34 \%$ \\
\hline & $26.1 \mathrm{~kb}$ & Incomplete & 22 & $3123500-3149610$ & PHAGE_Ralsto_RSY1_NC_025115(2) & $52.75 \%$ \\
\hline Dickeya paradisaica & $31.3 \mathrm{~kb}$ & Incomplete & 22 & $3944213-3975565$ & PHAGE_Paenib_Fern_NC_028851(1) & $48.84 \%$ \\
\hline NCPPB 2511 & $17.0 \mathrm{~kb}$ & Questionable & 22 & $4081106-4098183$ & PHAGE_Entero_P4_NC_001609(3) & $50.16 \%$ \\
\hline$\left(\mathrm{NZ} \_\mathrm{CM} 001857\right)^{* *}$ & $36.4 \mathrm{~kb}$ & Intact & 37 & $4377338-4413753$ & PHAGE_Salmon_SEN5_NC_028701(18) & $55.43 \%$ \\
\hline
\end{tabular}

a **, (.gff and .fasta); and $*$, (.fasta). 
TABLE 5. (Continued from previous page)

\begin{tabular}{|c|c|c|c|c|c|c|}
\hline Organism (accession) & $\begin{array}{c}\text { Region } \\
\text { length }(\mathrm{kb})\end{array}$ & Completeness & $\begin{array}{c}\text { Number of } \\
\text { proteins }\end{array}$ & Position & Phage (GenBank accession number) & $\mathrm{GC} \%$ \\
\hline \multirow{6}{*}{$\begin{array}{l}\text { Pectobacterium } \\
\text { carotovorum ssp. } \\
\text { carotovorum PC1 } \\
\left(\text { NC_012917)** }^{*}\right.\end{array}$} & $12.9 \mathrm{~kb}$ & Incomplete & 15 & $833155-845434$ & PHAGE_Entero_P4_NC_001609(5) & $46.86 \%$ \\
\hline & $20.9 \mathrm{~kb}$ & Incomplete & 24 & $1811972-1832962$ & PHAGE_Burkho_BcepMu_NēC_005882(16) & $53.15 \%$ \\
\hline & $13.8 \mathrm{~kb}$ & Incomplete & 17 & $2642964-2656853$ & PHAGE_Shigel_Ss_VASD_NC_028685(2) & $43.54 \%$ \\
\hline & $36.4 \mathrm{~kb}$ & Intact & 48 & $2986224-3022684$ & PHAGE_Salmon_SEN4_NC_029015(20) & $49.57 \%$ \\
\hline & $35.4 \mathrm{~kb}$ & Intact & 50 & $3573259-3608691$ & PHAGE_Entero_PsP3_NC_005340(18) & $52.08 \%$ \\
\hline & $12.8 \mathrm{~kb}$ & Incomplete & 15 & $3683594-3696452$ & PHAGE_Entero_P4_NC_001609(5) & $45.98 \%$ \\
\hline
\end{tabular}

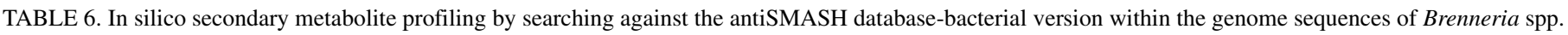
and relevant Pectobacteriaceae strains

\begin{tabular}{|c|c|c|c|c|}
\hline Organism (accession) & Type $^{\mathrm{a}}$ & Position & Most similar known cluster & Similarity $(\%)$ \\
\hline \multirow[t]{4}{*}{ B. izadpanahii (Iran 50) (CP050854) } & NRPS-T1PKS & $558,967-615,840$ & Yersiniabactin & $8 \%$ \\
\hline & Thiopeptide & $2,039,704-2,064,242$ & O-antigen & $14 \%$ \\
\hline & NRPS & $2,900,414-2,947,453$ & Turnerbactin & $38 \%$ \\
\hline & Thiopeptide-LAP & $3,151,259-3,205,833$ & Klebsazolicin & $60 \%$ \\
\hline \multirow[t]{4}{*}{ B. goodwinii FRB141 (CP014137) } & NRPS & $1,334,406-1,379,9911$ & Turnerbactin & $23 \%$ \\
\hline & Thiopeptide & $2,090,803-2,117,249$ & O-antigen & $14 \%$ \\
\hline & NRPS-T1PKS & $3,123,664-3,176,845$ & - & - \\
\hline & NRPS-T1PKS & $4,483,386-4,507,022$ & Yersiniabactin & $10 \%$ \\
\hline \multirow[t]{9}{*}{ B. goodwinii FRB171 (MJLY00000000) } & Thiopeptide & $240,768-267,250$ & O-antigen & $14 \%$ \\
\hline & NRPS-T1PKS & $43,419-98,435$ & - & - \\
\hline & Arylpolyene & $16,023-59,618$ & Arylpolyene & $100 \%$ \\
\hline & NRPS & $1-31,659$ & - & - \\
\hline & NRPS-T1PKS & $1-16,865$ & Microcin & $26 \%$ \\
\hline & NRPS & $37,233-65,550$ & Amonabactin & $42 \%$ \\
\hline & NRPS & $1-6,339$ & - & - \\
\hline & NRPS & $1-3173$ & - & - \\
\hline & NRPS & $1-3141$ & - & - \\
\hline \multirow[t]{6}{*}{ B. goodwinii OBR1 (CGIG00000000) } & NRPS-T1PKS & $523,740-575,858$ & - & - \\
\hline & Arylpolyene & $1,624,347-1,667,942$ & Arylpolyene & $100 \%$ \\
\hline & NRPS & $2,367,733-2,410,744$ & Thailanstatin & $10 \%$ \\
\hline & NRPS-T1PKS & $3,652,192-3,715,065$ & N-myristoyl-D-asparagine & $8 \%$ \\
\hline & NRPS & $4,052,101-4,099,132$ & Turnerbactin & $23 \%$ \\
\hline & Thiopeptide & $4,813,399-4,839,881$ & O-antigen & $14 \%$ \\
\hline \multirow[t]{7}{*}{ B. nigrifluens ATCC 13028 (CР034036) } & Betalactone & $749,459-773,280$ & - & - \\
\hline & Hserlactone & $739,318-805,205$ & Taxlllaid & $8 \%$ \\
\hline & NRPS-T1PKS & $2,202,105-2,260,740$ & Yersiniabactin & $14 \%$ \\
\hline & Thiopeptide & $2,746,000-2,772,417$ & O-antigen & $14 \%$ \\
\hline & NRPS & $2,945,000-3,030,660$ & Pseudomonine & $20 \%$ \\
\hline & NRPS-T1PKS & $4,169,935-4,261,339$ & Entrerobactin & $12 \%$ \\
\hline & Arylpolyene & $4,786,417-4,829,997$ & Arylpolyene & $100 \%$ \\
\hline \multirow[t]{8}{*}{ Brenneria sp. EniD312 (NZ_CM001230.1) } & Betalactone & $748,456-772,277$ & - & - \\
\hline & Hserlactone & $1,760,324-1,780,956$ & Taxlllaid & $8 \%$ \\
\hline & NRPS-T1PKS & $2,196,941-2,255,576$ & Yersiniabactin & $14 \%$ \\
\hline & Thiopeptide & $2,740,461-2,766,878$ & O-antigen & $14 \%$ \\
\hline & NRPS & $2,932,508-2,979,014$ & Pyoverdine & $1 \%$ \\
\hline & NRPS & $2,995,656-3,082,947$ & Pseudomonine & $20 \%$ \\
\hline & NRPS-T1PKS & $4,222,222-4,313,626$ & Entrerobactin & $12 \%$ \\
\hline & Arylpolyene & $4,838,702-4,882,282$ & Arylpolyene & $100 \%$ \\
\hline \multirow[t]{7}{*}{ B. alni NCPPB 3934 (MJLZ00000000) } & $\begin{array}{l}\text { Betalactone/ } \\
\text { siderophore }\end{array}$ & $72,368-100,981$ & Aerobactin & $100 \%$ \\
\hline & Phenaine & $99,314-119,772$ & Fontizine & $60 \%$ \\
\hline & NRPS & $3,616-59,328$ & Turnerbactin & $30 \%$ \\
\hline & Arylpolyene & $1,989-45,584$ & Arylpolyene & $88 \%$ \\
\hline & T1PKS & $1-42,595$ & Filipine & $15 \%$ \\
\hline & Ladderane & $1-29,330$ & - & - \\
\hline & NRPS-T1PKS & $9,487-47,918$ & - & - \\
\hline \multirow[t]{8}{*}{ B. salicis DSM30166 (MJMA00000000) } & $\begin{array}{l}\text { Betalactone/ } \\
\text { siderophore }\end{array}$ & $137,738-165,465$ & Aerobactin & $100 \%$ \\
\hline & Hserlactone & $1-18,735$ & - & - \\
\hline & NRPS & $64,241-90,327$ & - & - \\
\hline & NRPS & $517-56,210$ & Turnerbactin & $30 \%$ \\
\hline & Thiopeptide & $40,220-60,229$ & O-antigen & $14 \%$ \\
\hline & NRPS-T1PKS & $5,785-36,071$ & - & - \\
\hline & T1PKS & $1-25,905$ & Oronofacic acid & $100 \%$ \\
\hline & Arylpolyene & $1-17,535$ & - & - \\
\hline
\end{tabular}

(Continued on next page)

a NRPS, nonribosomal peptide synthetase cluster; LAP, linear azol (in)e-containing peptides; TPKS, type (I, II, or III) polyketide synthase; and hserlactone, homoserine lactone cluster. 
TABLE 6. (Continued from previous page)

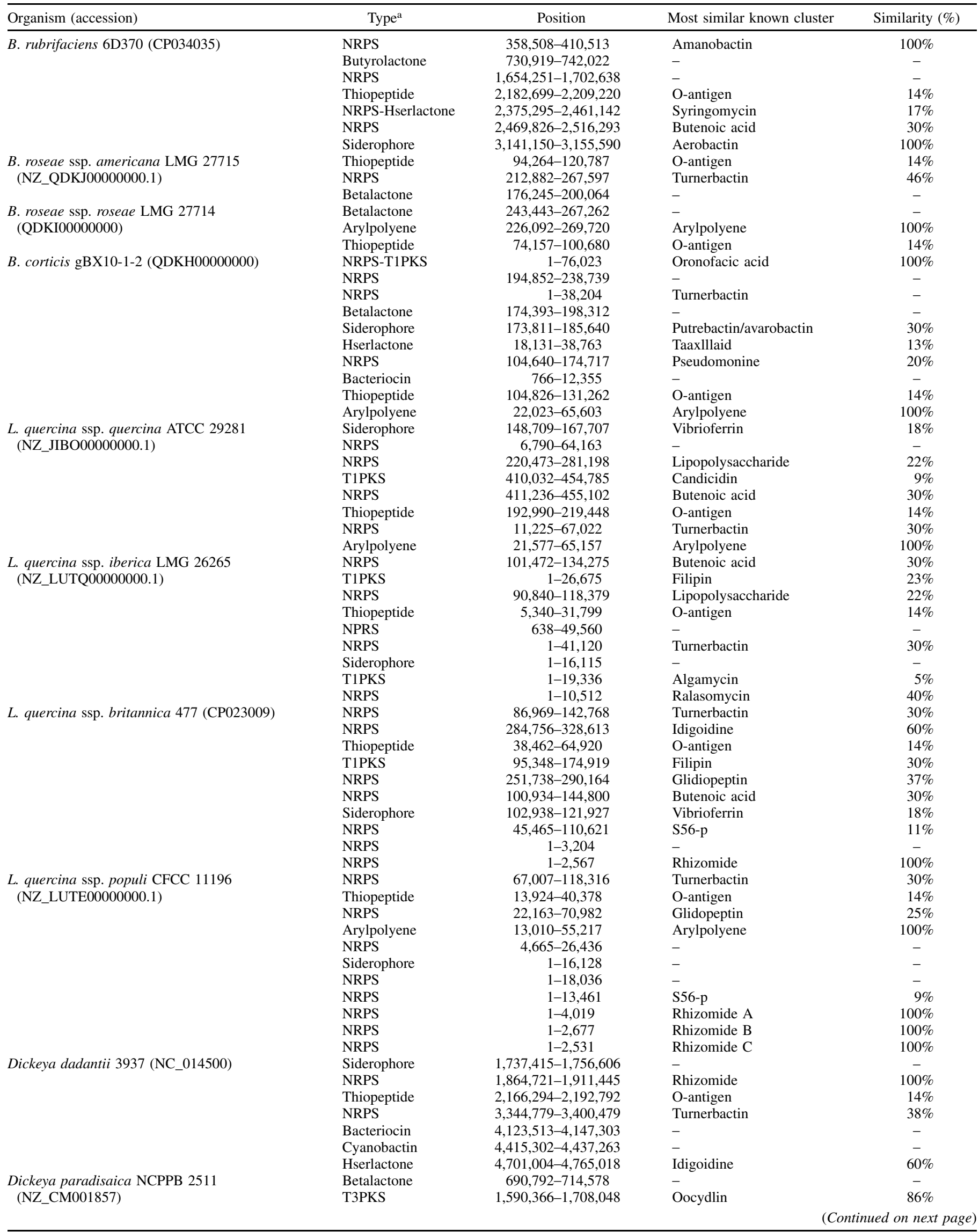


TABLE 6. (Continued from previous page)

\begin{tabular}{|c|c|c|c|c|}
\hline Organism (accession) & Type $^{\mathrm{a}}$ & Position & Most similar known cluster & Similarity $(\%)$ \\
\hline \multirow{11}{*}{$\begin{array}{l}\text { Pectobacterium carotovorum ssp. } \\
\text { carotovorum PC1 (NC_012917) }\end{array}$} & NRPS & $1,722,708-1,776,660$ & Amonabactin & $57 \%$ \\
\hline & NRPS & $1,944,874-1,999,802$ & Sulfazecin & $11 \%$ \\
\hline & Thiopeptide & $2,603,050-2,629,807$ & O-antigen & $14 \%$ \\
\hline & NRPS & $3,508,607-3,616,479$ & S56-p1 & $15 \%$ \\
\hline & Siderophore & $3,649,496-3,664,102$ & Vibrioferrin & $36 \%$ \\
\hline & NRPS & $508,574-562,953$ & Amonabactin & $100 \%$ \\
\hline & NRPS-T1PKS & $639,867-695,982$ & - & - \\
\hline & Thiopeptide & $1,985,432-2,011,857$ & O-antigen & $14 \%$ \\
\hline & Betalactone & $4,056,003-4,079,817$ & - & - \\
\hline & Siderophore & $4,390,324-4,402,225$ & - & - \\
\hline & Hserlactone & $4,668,638-4,689,288$ & - & - \\
\hline
\end{tabular}

species (B. goodwinii, B. roseae ssp. roseae, B. nigrifluens-only two isolates-, and Brenneria sp.-now named B. izadpanahii) from individual symptomatic oak trees indicated the complexity of the syndromes that Brenneria species are involved (Bakhshi ganje et al. 2020). In the light of high-throughput sequencing technologies and improved metagenomics (e.g., metagenomic based- strain-level identification [Mechan Llontop et al. 2020]), metatranscriptomics, and metaproteomics analyses, studies of tree and forest ecosystem microbiomes have increased and consequently lead to developing an understanding of complicated communications between the species.

Description of $B$. izadpanahii sp. nov. B. izadpanahii sp. nov. (i.zad.pa.nah'i.i. N.L. gen. n. izadpanahii, named in honor of Professor Keramatollah Izadpanah, for his 6 decades of efforts in plant pathology education and research in Iran). Cells are gram stain-negative and facultatively anaerobic. Colonies are pale cream, circular, opaque, convex with entire margins, smooth and approximately 0.5 to $1.0 \mathrm{~mm}$ in diameter after incubation for 2 days at $30^{\circ} \mathrm{C}$ on nutrient agar. Growth occurs at 10 to $40^{\circ} \mathrm{C}$ and the optimum growth temperature is $30^{\circ} \mathrm{C}$. It is negative for activities of lysine decarboxylase, arbutin ( $\beta$-galactosidase), arginine dihydrolase, nitrate reductase, ornithine decarboxylase, urease, oxidase, d-arabinose, and 1-rhamnose. It is positive for fermentation of inositol, amygdalin, sucrose, glucose, mannitol, and arabinose. Positive for acid production from glycerol, L-arabinose, D-xylose, aesculin ferric citrate, D-glucose, D-fructose, $\beta$-d-methyl glycoside, inositol, D-mannitol, raffinose, melibiose, sucrose, trehalose, and D-galactose. It is positive for assimilation of Tween $80, \mathrm{~N}$-acetylD-glucosamine, L-arabinose, D-fructose, D-galactose, a-D-glucose, m-inositol, D-mannitol, D-mannose, melibiose, raffinose, sucrose, trehalose, glycerol, glucose-1-phosphate, and glucose-6-phosphate. The DNA G+C content is $53.66 \mathrm{~mol} \%$. The type strain is Iran isolate 50 (BioProject PRJNA616088; accession number CP050854 = Iran 52 = Iran 29), isolated from symptomatic bark of $Q$. castaneifolia canker in north of Iran.

\section{LITERATURE CITED}

Adeniji, A. A., and Babalola, O. O. 2019. Bacillus velezensis: Phylogeny, useful applications, and avenues for exploitation. Appl. Microbiol. Biotechnol. 103:3669-3682.

Adeolu, M., Alnajar, S., Naushad, S., and Gupta, R. S. 2016. Genome-based phylogeny and taxonomy of the 'Enterobacteriales': Proposal for Enterobacterales ord. nov. divided into the families Enterobacteriaceae, Erwiniaceae fam. nov., Pectobacteriaceae fam. nov., Yersiniaceae fam. nov., Hafniaceae fam. nov., Morganellaceae fam. nov., and Budviciaceae fam. nov. Int. J. Syst. Evol. Microbiol. 66:5575-5599.

Arkin, A. P., Cottingham, R. W., Henry, C. S., Harris, N. L., Stevens, R. L., Maslov, S., Dehal, P., Ware, D., Perez, F., Canon, S., and Sneddon, M. W. 2018. KBase: The United States Department of Energy Systems Biology Knowledgebase. Nat. Biotechnol. 36:566-569.

Arndt, D., Grant, J. R., Marcu, A., Sajed, T., Pon, A., Liang, Y., and Wishart, D. S. 2016. PHASTER: A better, faster version of the PHAST phage search tool. Nucleic Acids Res. 44:W16-W21.

Arnold, D. L., and Jackson, R. W. 2011. Bacterial genomes: Evolution of pathogenicity. Curr. Opin. Plant Biol. 14:385-391.
Bakhshi ganje, M., Shams-Bakhsh, M., Mackay, J., and Rahimian, H. 2020. Identification and characterization of bacterial strains associated with diseased oak trees in Northern Iran. For. Pathol. 50:e12571.

Bertelli, C., Laird, M. R., Williams, K. P., Simon Fraser University Research Computing Group, Lau, B. Y., Hoad, G., Winsor, G. L., and Brinkman, F. S. L. 2017. IslandViewer 4: Expanded prediction of genomic islands for larger-scale datasets. Nucleic Acids Res. 45:W30-W35.

Blin, K., Shaw, S., Steinke, K., Villebro, R., Ziemert, N., Lee, S. Y., Medema, M. H., and Weber, T. 2019. antiSMASH 5.0: Updates to the secondary metabolite genome mining pipeline. Nucleic Acids Res. 47:W81-W87.

Brady, C., Cleenwerck, I., Venter, S., Vancanneyt, M., Swings, J., and Coutinho, T. 2008. Phylogeny and identification of Pantoea species associated with plants, humans and the natural environment based on multilocus sequence analysis (MLSA). Syst. Appl. Microbiol. 31:447-460.

Brady, C., Hunter, G., Kirk, S., Arnold, D., and Denman, S. 2014. Description of Brenneria roseae sp. nov. and two subspecies, Brenneria roseae subspecies roseae ssp. nov. and Brenneria roseae subspecies americana ssp. nov. isolated from symptomatic oak. Syst. Appl. Microbiol. 37:396-401.

Brady, C. L., Cleenwerck, I., Denman, S., Venter, S. N., Rodríguez-Palenzuela, P., Coutinho, T. A., and De Vos, P. 2012. Proposal to reclassify Brenneria quercina (Hildebrand and Schroth 1967) Hauben et al. 1999 into a new genus, Lonsdalea gen. nov., as Lonsdalea quercina comb. nov., descriptions of Lonsdalea quercina subsp. quercina comb. nov., Lonsdalea quercina subsp. iberica subsp. nov. and Lonsdalea quercina subsp. britannica subsp. nov., emendation of the description of the genus Brenneria, reclassification of Dickeya dieffenbachiae as Dickeya dadantii subsp. dieffenbachiae comb. nov., and emendation of the description of Dickeya dadantii. Int. J. Syst. Evol. Microbiol. 62:1592-1602.

Broberg, M., Doonan, J., Mundt, F., Denman, S., and McDonald, J. E. 2018. Integrated multi-omic analysis of host-microbiota interactions in acute oak decline. Microbiome 6:21.

Caballero, J. I., Zerillo, M. M., Snelling, J., Cranshaw, W., Boucher, C., and Tisserat, N. 2014. Genome sequences of strain ATCC 29281 and pin and northern red oak isolates of Lonsdalea quercina subsp. quercina. Genome Announc. 2(3).

Caputo, A., Fournier, P. E., and Raoult, D. 2019. Genome and pan-genome analysis to classify emerging bacteria. Biol. Direct 14:5.

Carattoli, A., Zankari, E., Garcìa-Fernandez, A., Larsen, M. V., Lund, O., Villa, L., Aarestrup, F. M., and Hasman, H. 2014. In silico detection and typing of plasmids using PlasmidFinder and plasmid multilocus sequence typing. Antimicrobiol. Agents Chemother. 58: 3895-3903.

Czajkowski, R. 2019. May the phage be with you? Prophage-like elements in the genomes of soft rot Pectobacteriaceae: Pectobacterium spp. and Dickeya spp. Front. Microbiol. 10:138.

Denman, S., Brady, C., Kirk, S., Cleenwerck, I., Venter, S., Coutinho, T., and De Vos, P. 2012. Brenneria goodwinii sp. nov., associated with acute oak decline in the UK. Int. J. Syst. Evol. Microbiol. 62:2451-2456.

Doonan, J., Denman, S., Pachebat, J. A., and McDonald, J. E. 2019. Genomic analysis of bacteria in the acute oak decline pathobiome. Microbiol. Genom. 5:1-15.

Evans, T. J., Coulthurst, S. J., Komitopoulou, E., and Salmond, G. P. 2010. Two mobile Pectobacterium atrosepticum prophages modulate virulence. FEMS Microbiol. Lett. 304:195-202.

Glasner, J. D., Yang, C. H., Reverchon, S., Hugouvieux-Cotte-Pattat, N., Condemine, G., Bohin, J. P., Van Gijsegem, F., Yang, S., Franza, T., Expert, D., and Plunkett, G. 2011. Genome sequence of the plant-pathogenic bacterium Dickeya dadantii 3937. J. Bacteriol. 193:2076-2077.

Glazebrook, J. 2005. Contrasting mechanisms of defense against biotrophic and necrotrophic pathogens. Annu. Rev. Phytopathol. 43:205-227.

Grammbitter, G. L., Schmalhofer, M., Karimi, K., Shi, Y. M., Schöner, T. A., Tobias, N. J., Morgner, N., Groll, M., and Bode, H. B. 2019. An uncommon 
type II PKS catalyzes biosynthesis of aryl polyene pigments. J. Am. Chem. Soc. 141:16615-16623.

Han, A. W., Sandy, M., Fishman, B., Trindade-Silva, A. E., Soares, C. A., Distel, D. L., Butler, A., and Haygood, M. G. 2013. Turnerbactin, a novel triscatecholate siderophore from the shipworm endosymbiont Teredinibacter turnerae T7901. PLoS One 8:e76151.

Hauben, L., Moore, E. R., Vauterin, L., Steenackers, M., Mergaert, J., Verdonck, L., and Swings, J. 1998. Phylogenetic position of phytopathogens within the Enterobacteriaceae. Syst. Appl. Microbiol. 21:384-397.

Hulin, M. T., Armitage, A. D., Vicente, J. G., Holub, E. B., Baxter, L., Bates, H. J., Mansfield, J. W., Jackson, R. W., and Harrison, R. J. 2018. Comparative genomics of Pseudomonas syringae reveals convergent gene gain and loss associated with specialization onto cherry (Prunus avium). New Phytol. 219:672-696.

Kaczmarek, M., Mullett, M. S., McDonald, J. E., and Denman, S. 2017. Multilocus sequence typing provides insights into the population structure and evolutionary potential of Brenneria goodwinii, associated with acute oak decline. PLoS One 12:e0178390.

Lee, I., Kim, Y. O., Park, S. C., and Chun, J. 2016. OrthoANI: An improved algorithm and software for calculating average nucleotide identity. Intl. J. Syst. Evol. Microbiol. 66:1100-1103.

Lee, Y. A., Yang, P. Y., and Huang, S. C. 2020. Characterization, phylogeny, and genome analyses of nonpathogenic Xanthomonas campestris strains isolated from brassica seeds. Phytopathology 110:981-988.

Li, Y., Fang, W., Xue, H., Liang, W. X., Wang, L. F., Tian, G. Z., and Piao, C. G. 2015. Brenneria populi sp. nov., isolated from symptomatic bark of Populus $\times$ euramericana canker. Int. J. Syst. Evol. Microbiol. 65:432-437.

Li, Y., Zheng, M. H., Wang, H. M., Lin, C. L., and Wang, X. Z. 2019. Brenneria corticis sp. nov., isolated from symptomatic bark of Populus $\times$ euramericana canker. Intl. J. Syst. Evol. Microbiol. 69:63-67.

Lombard, V., Golaconda Ramulu, H., Drula, E., Coutinho, P. M., and Henrissat, B. 2014. The carbohydrate-active enzymes database (CAZy) in 2013. Nucleic Acids Res. 42:D490-D495.

Maes, M., Huvenne, H., and Messens, E. 2009. Brenneria salicis, the bacterium causing watermark disease in willow, resides as an endophyte in wood. Environ. Microbiol. 11:1453-1462.

Matos, R. C., Lapaque, N., Rigottier-Gois, L., Debarbieux, L., Meylheuc, T., Gonzalez-Zorn, B., Repoila, F., de Fatima Lopes, M., and Serror, P. 2013. Enterococcus faecalis prophage dynamics and contributions to pathogenic traits. PLoS Genet. 9:e1003539.

Mechan Llontop, M. E., Sharma, P., Aguilera Flores, M., Yang, S., Pollok, J., Tian, L., Huang, C., Rideout, S., Heath, L. S., Li, S., and Vinatzer, B. A. 2020. Strain-level identification of bacterial tomato pathogens directly from metagenomic sequences. Phytopathology 110:768-779.

Meier-Kolthoff, J. P., Auch, A. F., Klenk, H. P., and Göker, M. 2013. Genome sequence-based species delimitation with confidence intervals and improved distance functions. BMC Bioinform. 14:60.

Niemann, S., Pühler, A., Tichy, H. V., Simon, R., and Selbitschka, W. 1997. Evaluation of the resolving power of three different DNA fingerprinting methods to discriminate among isolates of a natural Rhizobium meliloti population. J. Appl. Microbiol. 82:477-484.

Ohnishi, M., Kurokawa, K., and Hayashi, T. 2001. Diversification of Escherichia coli genomes: Are bacteriophages the major contributors? Trends Microbiol. 9:481-485.

Overbeek, R., Olson, R., Pusch, G. D., Olsen, G. J., Davis, J. J., Disz, T., Edwards, R. A., Gerdes, S., Parrello, B., Shukla, M., and Vonstein, V. 2014. The SEED and the rapid annotation of microbial genomes using subsystems technology (RAST). Nucleic Acids Res. 42:D206-D214.
Parks, D. H., Imelfort, M., Skennerton, C. T., Hugenholtz, P., and Tyson, G. W. 2015. CheckM: Assessing the quality of microbial genomes recovered from isolates, single cells, and metagenomes. Genome Res. 25:1043-1055.

Pérombelon, M. C. M. 2002. Potato diseases caused by soft rot Erwinias: An overview of pathogenesis. Plant Pathol. 51:1-12.

Pettifor, B. J., Doonan, J., Denman, S., and McDonald, J. E. 2020. Survival of Brenneria goodwinii and Gibbsiella quercinecans, associated with acute oak decline, in rainwater and forest soil. Syst. Appl. Microbiol. 43: 126052.

Poret-Peterson, A. T., McClean, A. E., Chen, L., and Kluepfel, D. A. 2019. Complete genome sequences of Brenneria rubrifaciens strain 6D370 and Brenneria nigrifluens strain ATCC 13028, causative agents of bark cankers in walnut. Microbiol. Resour. Announc. 8:e00597-19.

Price, M. N., Dehal, P. S., and Arkin, A. P. 2010. FastTree 2-Approximately maximum-likelihood trees for large alignments. PLoS One 5:e9490.

Pritchard, L., Humphris, S., Saddler, G. S., Elphinstone, J. G., Pirhonen, M., and Toth, I. K. 2013. Draft genome sequences of 17 isolates of the plant pathogenic bacterium Dickeya. Genome Announc. 26:1(6).

Richter, M., Rosselló-Móra, R., Oliver Glöckner, F., and Peplies, J. 2016. JSpeciesWS: A web server for prokaryotic species circumscription based on pairwise genome comparison. Bioinformatics 32:929-931.

Sapp, M., Lewis, E., Moss, S., Barrett, B., Kirk, S., Elphinstone, J. G., and Denman, S. 2016. Metabarcoding of bacteria associated with the acute oak decline syndrome in England. Forests 7:95.

Tampakaki, A. P., Skandalis, N., Gazi, A. D., Bastaki, M. N., Panagiotis, F. S., Charova, S. N., Kokkinidis, M., and Panopoulos, N. J. 2010. Playing the "Harp": Evolution of our understanding of hrp/hrc genes. Annu. Rev. Phytopathol. 48:347-370.

Toth, I. K., Pritchard, L., and Birch, P. R. J. 2006. Comparative genomics reveals what makes an enterobacterial plant pathogen. Annu. Rev. Phytopathol. 44:305-336.

Vorholt, J. A. 2012. Microbial life in the phyllosphere. Nat. Rev. Microbiol. 10:828-840.

Wattam, A. R., Davis, J. J., Assaf, R., Boisvert, S., Brettin, T., Bun, C., Conrad, N., Dietrich, E. M., Disz, T., Gabbard, J. L., and Gerdes, S. 2017. Improvements to PATRIC, the all-bacterial bioinformatics database and analysis resource center. Nucleic Acids Res. 45:D535-D542.

Winstanley, C., and Hart, C. A. 2001. Type III secretion systems and pathogenicity islands. J. Med. Microbiol. 50:116-126.

Xu, L., Dong, Z., Fang, L., Luo, Y., Wei, Z., Guo, H., Zhang, G., Gu, Y. Q., Coleman-Derr, D., Xia, Q., and Wang, Y. 2019. OrthoVenn2: A web server for whole-genome comparison and annotation of orthologous clusters across multiple species. Nucleic Acids Res. 47:W52-W58.

Yoon, S. H., Ha, S. M., Lim, J. M., Kwon, S. J., and Chun, J. 2017. A largescale evaluation of algorithms to calculate average nucleotide identity. Antonie van Leeuwenhoek 110:1281-1286.

Zhang, H., Yohe, T., Huang, L., Entwistle, S., Wu, P., Yang, Z., Busk, P. K., $\mathrm{Xu}$, Y., and Yin, Y. 2018. dbCAN2: A meta server for automated carbohydrate-active enzyme annotation. Nucleic Acids Res. 46:W95W101.

Zhang, Y., Fan, Q., and Loria, R. 2016. A re-evaluation of the taxonomy of phytopathogenic genera Dickeya and Pectobacterium using whole-genome sequencing data. Syst. Appl. Microbiol. 39:252-259.

Zheng, M. H., Piao, C. G., Xue, H., Guo, M. W., and Li, Y. 2017. Brenneria populi subsp. brevivirga subsp. nov. isolated from symptomatic bark of Populus $\times$ euramericana canker, and description of Brenneria populi subsp. populi subsp. nov. Int. J. Syst. Evol. Microbiol. 67:3633-3638. 